TRANSACTIONS OF THE

AMERICAN MATHEMATICAL SOCIETY

Volume 358, Number 5, Pages 2123-2159

S 0002-9947(05)03740-2

Article electronically published on May 9, 2005

\title{
SECOND-ORDER HYPERBOLIC S.P.D.E.'S DRIVEN BY HOMOGENEOUS GAUSSIAN NOISE ON A HYPERPLANE
}

\author{
ROBERT C. DALANG AND OLIVIER LÉVÊQUE
}

\begin{abstract}
We study a class of hyperbolic stochastic partial differential equations in Euclidean space, that includes the wave equation and the telegraph equation, driven by Gaussian noise concentrated on a hyperplane. The noise is assumed to be white in time but spatially homogeneous within the hyperplane. Two natural notions of solutions are function-valued solutions and random field solutions. For the linear form of the equations, we identify the necessary and sufficient condition on the spectral measure of the spatial covariance for existence of each type of solution, and it turns out that the conditions differ. In spatial dimensions 2 and 3 , under the condition for existence of a random field solution to the linear form of the equation, we prove existence and uniqueness of a random field solution to non-linear forms of the equation.
\end{abstract}

\section{INTRODUCTION}

Stochastic partial differential equations (s.p.d.e.'s) driven by spatially homogeneous Gaussian noise that is white in time, first studied in the parabolic case by Dawson and Salehi $[8$, has recently been the subject of several papers, beginning with the work of Mueller 22 and Dalang and Frangos [7] on the wave equation in two spatial dimensions, and continued in several recent papers, such as Dalang [5], Millet and Sanz-Solé 20, 21, Peszat 25] and Peszat and Zabczyk [26.

In this paper, we are interested in equations that might arise when modelling situations such as the following. Rain falls on the surface of a lake, producing a sound wave that propagates under water. This noise is produced by a large number of small contributions (the rain droplets). After suitable rescaling, the noise can be considered to be spatially homogeneous on the surface of the lake, propagating through a three-dimensional medium. Hence, the noise is concentrated on the twodimensional boundary of a three-dimensional domain.

There have been several studies of equations driven by noise concentrated on manifolds. The noise is generally taken to be a stochastic boundary condition. Many of these are carried out in spatial dimension one, so the boundary noise is a pointwise noise, as in 2, 10, 18. There are also results for parabolic s.p.d.e.'s in

Received by the editors January 26, 2004 and, in revised form, May 4, 2004.

2000 Mathematics Subject Classification. Primary 60H15; Secondary 60G15, 35R60.

Key words and phrases. Stochastic partial differential equations, spatially homogeneous Gaussian noise, hyperbolic equations.

The research of the first author was partially supported by the Swiss National Foundation for Scientific Research.

This article is based on part of the second author's Ph.D. thesis, written under the supervision of the first author. 
higher dimensions [19, 29]. Since parabolic equations exhibit regularizing properties, the noise can be taken to be concentrated on a rather general manifold, but there is no reason to expect that similar methods or results will apply to hyperbolic equations, for which the results are quite different (see Remark 4.10).

In this paper, we fix $a, b \in \mathbb{R}$ and consider the following non-linear s.p.d.e. and some generalizations of it:

$$
\left\{\begin{aligned}
\frac{\partial^{2} u}{\partial t^{2}}(t, x) & +2 a \frac{\partial u}{\partial t}(t, x)+b u(t, x)-\Delta u(t, x) \\
+ & =g\left(u\left(t, x_{1}, 0\right)\right) \delta_{0}\left(x_{2}\right) \\
+ & h\left(u\left(t, x_{1}, 0\right)\right) \dot{F}\left(t, x_{1}\right) \delta_{0}\left(x_{2}\right), \quad t \in \mathbb{R}_{+}, x=\left(x_{1}, x_{2}\right) \in \mathbb{R}^{d-1} \times \mathbb{R}
\end{aligned}\right.
$$

where $g$ and $h$ are real-valued functions satisfying standard conditions and $\dot{F}$ is a Gaussian noise whose covariance is formally given by

$$
\mathbb{E}\left(\dot{F}\left(t, x_{1}\right) \dot{F}\left(s, y_{1}\right)\right)=\delta_{0}(t-s) \Gamma\left(x_{1}-y_{1}\right) .
$$

Note that the variable $x_{1}$ represents coordinates in the hyperplane $\mathbb{R}^{d-1} \times\{0\}$ on which the noise is concentrated, and $x_{2}$ is the coordinate in the direction perpendicular to this hyperplane.

There are at least three interesting special cases of this equation. When $a=b=$ 0 , this is the wave equation. When $a>0$ and $b=0$, this is the wave equation with damping, also called the telegraph equation when $d=1$. And finally, when $a=0$ and $b \neq 0$, this is the Klein-Gordon equation.

Given the irregularity of the noise, the first issue is to give a rigorous meaning to this equation. We do this via Walsh's theory of martingale measures [30, using an appropriate extension of his stochastic integral, in the spirit of [5]. The linear case $g \equiv 0$ and $h \equiv 1$ is already of considerable interest. Indeed, it is only in spatial dimension 1 that equation (1.1) will have a real-valued solution for all choices of the covariance $\Gamma$; in higher dimensions, the solution exists in general only in the space of random (Schwartz) distributions. Our first objective is to characterize those covariances for which the linear form of (1.1) has a real-valued solution.

In this context, a surprising distinction appears. Indeed, there are at least two natural ways to define real-valued solutions. One of these is the notion of functionvalued solution (see Section 4.1), in which, essentially, the solution is at each time $t$ a random function of the space variable $x$. Another is the notion of random field solution (see Section 4.2), in which the solution is a random field defined for every $(t, x) \in \mathbb{R}_{+} \times \mathbb{R}^{d}$, with an $L^{2}$-continuity requirement. It turns out that these notions are distinct, and we give in Theorems 4.5 and 4.8 respectively, the necessary and sufficient conditions on the spectral measure of the covariance for existence of either notion of solution.

In the case where the linear form of equation (1.1) has a random field solution, it is natural to consider the non-linear form of the equation. We show in Theorem 5.1 that in spatial dimensions 2 and 3, the non-linear equation has a solution under the same conditions on the covariance as those obtained for the linear equation.

The outline of this paper is as follows. In Section 2, we consider equation (1.1) with a slightly more general form of driving noise, which contains the situation of noise concentrated on a hyperplane, and we construct an extension of Walsh's martingale measure stochastic integral following [5]. In Section 3, we analyze the Green's function of the equation (which is in fact not a function in dimensions 
greater than 2) and in particular, the integrability properties of its Fourier transform in the $x_{1}$-coordinates. These somewhat technical estimates are the key to the results of Section 4 in which we establish the necessary and sufficient conditions on the spectral measure of the noise for existence of the two kinds of real-valued solutions. In Section [5] we establish existence and uniqueness for the non-linear form of the equation. Finally, three appendices provide some background information: Appendix A contains a reformulation of the conditions on the spectral measure directly in terms of the covariance function, following the approaches of [17, 27]; Appendix B contains explicit formulas for the Green's function of the equation in spatial dimensions 2 and 3, and Appendix C recalls the properties of Bessel functions that are needed in this paper. For the reader's convenience, an index of notation is provided in Appendix D.

\section{A linear equation Driven by Gaussian noise}

Let $a, b \in \mathbb{R}$. We consider the following linear form of (1.1): (2.1)

$$
\left\{\begin{array}{l}
\frac{\partial^{2} u}{\partial t^{2}}(t, x)+2 a \frac{\partial u}{\partial t}(t, x)+b u(t, x)-\Delta u(t, x)=\dot{F}^{0}(t, x), \quad(t, x) \in \mathbb{R}_{+} \times \mathbb{R}^{d}, \\
u(0, x)=u_{0}(x), \quad \frac{\partial u}{\partial t}(0, x)=v_{0}(x), \quad x \in \mathbb{R}^{d},
\end{array}\right.
$$

where $u_{0}, v_{0}$ are two given (Schwartz) distributions on $\mathbb{R}^{d}$ and $\dot{F}^{0}=\left\{\dot{F}^{0}(t, x),(t, x)\right.$ $\left.\in \mathbb{R}_{+} \times \mathbb{R}^{d}\right\}$ is a generalized centered Gaussian process whose covariance is informally given by

$$
\mathbb{E}\left(\dot{F}^{0}(t, x) \dot{F}^{0}(s, y)\right)=\delta_{0}(t-s) \Gamma_{0}(x, y),
$$

where $\delta_{0}$ is the standard Dirac measure on $\mathbb{R}$ and $\Gamma_{0}$ is a non-negative definite measure on $\mathbb{R}^{d} \times \mathbb{R}^{d}$, in a sense that will be made precise below.

In order to give a meaning to this equation, several preliminaries are necessary.

2.1. Preliminaries. Fix a positive integer $d$. Following Schwartz 28$]$, let $\mathcal{S}\left(\mathbb{R}^{d}\right)$ be the space of complex-valued $C^{\infty}$-functions on $\mathbb{R}^{d}$ with rapid decrease, let $O_{M}\left(\mathbb{R}^{d}\right)$ be the space of complex-valued $C^{\infty}$ functions on $\mathbb{R}^{d}$ with polynomial growth, $\mathcal{S}^{\prime}\left(\mathbb{R}^{d}\right)$ be the space of tempered distributions on $\mathbb{R}^{d}$ (the dual of $\mathcal{S}\left(\mathbb{R}^{d}\right)$ ), and let $O_{C}^{\prime}\left(\mathbb{R}^{d}\right)$ be the space of distributions with rapid decrease on $\mathbb{R}^{d}$ (this is not the dual of $\left.O_{M}\left(\mathbb{R}^{d}\right)\right)$. The duality form between $T \in \mathcal{S}^{\prime}\left(\mathbb{R}^{d}\right)$ and $\varphi \in \mathcal{S}\left(\mathbb{R}^{d}\right)$ is denoted $\langle T, \varphi\rangle$. The Fourier transform $\mathcal{F} \varphi$ of $\varphi \in \mathcal{S}\left(\mathbb{R}^{d}\right)$ is defined by

$$
\mathcal{F} \varphi(\xi)=\int_{\mathbb{R}^{d}} d x \varphi(x) e^{i \xi \cdot x}, \quad \xi \in \mathbb{R}^{d}
$$

and the Fourier inversion formula reads

$$
\mathcal{F}^{-1} \varphi(\xi)=\frac{1}{(2 \pi)^{d}} \mathcal{F} \varphi(-\xi), \quad \xi \in \mathbb{R}^{d} .
$$

We will also use the notation $\mathcal{F T}$ for the Fourier transform of a tempered distribution $T \in \mathcal{S}^{\prime}\left(\mathbb{R}^{d}\right)$. By [28, Chap. VII, Thm XV],

$$
T \in O_{C}^{\prime}\left(\mathbb{R}^{d}\right) \text { if and only if } \mathcal{F} T \in O_{M}\left(\mathbb{R}^{d}\right) .
$$


For $\xi \in \mathbb{R}^{d}$, we denote by $\delta_{\xi}$ the standard Dirac measure at point $\xi$ and $\chi_{\xi}$ the function defined by $\chi_{\xi}(x)=e^{i \xi \cdot x}, x \in \mathbb{R}^{d}$ (note that $\mathcal{F} \delta_{\xi}=\chi_{\xi}$, so $\mathcal{F} \chi_{\xi}=(2 \pi)^{d} \delta_{-\xi}$ by (2.2) $)$.

2.2. Gaussian noise. In order to rigorously define the noise process $F^{0}$, we assume that $\Gamma_{0}$ is a signed Borel measure on $\mathbb{R}^{d} \times \mathbb{R}^{d}$ (with total variation measure $\left|\Gamma_{0}\right|$ ) which is non-negative definite on $\mathbb{R}^{d} \times \mathbb{R}^{d}$, that is,

$\sum_{i, j=1}^{m} c_{i} \overline{c_{j}} \Gamma_{0}\left(A_{i} \times A_{j}\right) \geq 0, \quad$ for all $m \geq 1, c_{1}, \ldots, c_{m} \in \mathbb{C}, A_{1}, \ldots, A_{m} \in \mathcal{B}_{b}\left(\mathbb{R}^{d}\right)$,

where $\mathcal{B}_{b}\left(\mathbb{R}^{d}\right)$ denotes the set of bounded Borel subsets of $\mathbb{R}^{d}$. In particular, $\Gamma_{0}(\cdot, \cdot)$ is symmetric (see [4, p. 68]). Furthermore, we assume that there exists a nonnegative Borel measure $\nu_{0}$ on $\mathbb{R}^{d} \times \mathbb{R}^{d}$, which is also non-negative definite, which dominates $\left|\Gamma_{0}\right|$, that is,

$$
\left|\Gamma_{0}\right|(A \times B) \leq \nu_{0}(A \times B), \quad \text { for all } A, B \in \mathcal{B}_{b}\left(\mathbb{R}^{d}\right),
$$

and which is tempered, that is, there exists $r>0$ such that

$$
\int_{\mathbb{R}^{d} \times \mathbb{R}^{d}} \frac{\nu_{0}(d x, d y)}{(1+|x|+|y|)^{r}}<\infty .
$$

Note that in general, $\left|\Gamma_{0}\right|$ is not non-negative definite even if $\Gamma_{0}$ is.

By the Kolmogorov extension theorem (see [23, Prop. 3.4]), there exists a probability space $(\Omega, \mathcal{G}, \mathbb{P})$ and a centered Gaussian process $F^{0}=\left\{F_{t}^{0}(\varphi), t \in\right.$ $\left.\mathbb{R}_{+}, \varphi \in \mathcal{S}\left(\mathbb{R}^{d}\right)\right\}$ defined on this space, whose covariance is given, for all $t, s \in \mathbb{R}_{+}$, $\varphi, \psi \in \mathcal{S}\left(\mathbb{R}^{d}\right)$, by

$$
\mathbb{E}\left(F_{t}^{0}(\varphi) \overline{F_{s}^{0}(\psi)}\right)=(t \wedge s) \int_{\mathbb{R}^{d} \times \mathbb{R}^{d}} \Gamma_{0}(d x, d y) \varphi(x) \overline{\psi(y)} .
$$

The Gaussian field $F^{0}$ is informally related to the noise $\dot{F}^{0}(t, x)$ in (2.1) by the formula

$$
F_{t}^{0}(\varphi)=\int_{0}^{t} d s \int_{\mathbb{R}^{d}} d x \dot{F}^{0}(s, x) \varphi(x), \quad t \in \mathbb{R}_{+}, \varphi \in \mathcal{S}\left(\mathbb{R}^{d}\right) .
$$

2.3. Stochastic integral. Since equation (2.1) is linear, it always has a solution in a space of Schwartz distributions. In order to get an explicit expression for this solution, we shall define a stochastic integral with respect to $F^{0}$. This section refers directly to [30, Chap. 2] and [7, 5, so some details will be omitted. Consider the filtration

$$
\mathcal{G}_{t}^{0}=\sigma\left\{F_{s}^{0}(\varphi), s \in[0, t], \varphi \in \mathcal{S}\left(\mathbb{R}^{d}\right)\right\} \vee \mathcal{N}, \quad t \in \mathbb{R}_{+},
$$

where $\mathcal{N}$ is the class of $\mathbb{P}$-null sets in $\Omega$. As in [7, the field $F^{0}$ extends to a worthy martingale measure $M^{0}=\left\{M_{t}^{0}(A), \mathcal{G}_{t}^{0}, t \in \mathbb{R}_{+}, A \in \mathcal{B}_{b}\left(\mathbb{R}^{d}\right)\right\}$ (see [30. Chap. 2] for a precise definition) with covariation measure $Q_{0}$ and dominating measure $K_{0}$ given respectively by

$$
Q_{0}([0, t] \times A \times B)=t \Gamma_{0}(A \times B) \quad \text { and } \quad K_{0}([0, t] \times A \times B)=t \nu_{0}(A \times B),
$$

for $t \in \mathbb{R}_{+}$and $A, B \in \mathcal{B}_{b}\left(\mathbb{R}^{d}\right)$. Further,

$$
F_{t}^{0}(\varphi)=\int_{[0, t] \times \mathbb{R}^{d}} \varphi(x) M^{0}(d s, d x) .
$$


Let $\mathcal{E}^{0}$ be the space of elementary integrands, that is, functions $\phi: \mathbb{R}_{+} \times \mathbb{R}^{d} \times \Omega \rightarrow$ $\mathbb{C}$ such that

$$
\phi(t, x, \omega)=1_{] a, b]}(t) 1_{A}(x) X(\omega),
$$

where $0 \leq a \leq b, A \in \mathcal{B}_{b}\left(\mathbb{R}^{d}\right)$ and $X$ is a bounded $\mathcal{G}_{a}^{0}$-measurable random variable. For such an element $\phi$ of $\mathcal{E}^{0}$, its stochastic integral with respect to the martingale measure $M^{0}$ is defined by

$$
\left(\phi \cdot M^{0}\right)_{t}(B)=X\left(M_{t \wedge b}^{0}(A \cap B)-M_{t \wedge a}^{0}(A \cap B)\right), \quad t \in \mathbb{R}_{+}, B \in \mathcal{B}_{b}\left(\mathbb{R}^{d}\right) .
$$

One easily checks the following isometry property:

$$
\mathbb{E}\left(\left(\phi \cdot M^{0}\right)_{t}(B) \overline{\left(\psi \cdot M^{0}\right)_{t}(C)}\right)=\left\langle\phi 1_{B}, \psi 1_{C}\right\rangle_{t, 0},
$$

for all $\phi, \psi \in \mathcal{E}^{0}, B, C \in \mathcal{B}_{b}\left(\mathbb{R}^{d}\right)$, where

$$
\left\langle\phi 1_{B}, \psi 1_{C}\right\rangle_{t, 0}=\mathbb{E}\left(\int_{0}^{t} d s \int_{B \times C} \Gamma_{0}(d x, d y) \phi(s, x) \overline{\psi(s, y)}\right) .
$$

We denote by $\|\cdot\|_{t, 0}$ the semi-norm induced by the semi-inner product $\langle\cdot, \cdot\rangle_{t, 0}$.

Let $\mathcal{P}^{0}$ be the predictable $\sigma$-field generated by the functions of $\mathcal{E}^{0}$, and term predictable functions those functions which are $\mathcal{P}^{0}$-measurable. For $t \in \mathbb{R}_{+}$and predictable $\phi:[0, t] \times \mathbb{R}^{d} \times \Omega \rightarrow \mathbb{C}$, let us define

$$
\|\phi\|_{t,+, 0}^{2}=\mathbb{E}\left(\int_{0}^{t} d s \int_{\mathbb{R}^{d} \times \mathbb{R}^{d}} \nu_{0}(d x, d y)|\phi(s, x) \phi(s, y)|\right) .
$$

Moreover, set

$$
H_{t,+, 0}=\left\{\phi:[0, t] \times \mathbb{R}^{d} \times \Omega \rightarrow \mathbb{C}: \phi \text { is predictable and }\|\phi\|_{t,+, 0}<\infty\right\} .
$$

It is well known (see [30, Chap. 2]) that the stochastic integral $\left(\phi \cdot M^{0}\right)_{t}(B)$ extends to elements of $H_{t,+, 0}$, in such a way that the isometry property (2.6) remains satisfied. In the following, we will adopt the notation $\left(\phi \cdot M^{0}\right)_{t}=\left(\phi \cdot M^{0}\right)_{t}\left(\mathbb{R}^{d}\right)$.

Note that for a deterministic integrand $\phi$, the stochastic integral process $\left(\phi \cdot M^{0}\right)=\left\{\left(\phi \cdot M^{0}\right)_{t}, t \in \mathbb{R}_{+}\right\}$is a Gaussian process. Furthermore, for deterministic integrands $\phi$ and $\psi$, the isometry property becomes

$$
\mathbb{E}\left(\left(\phi \cdot M^{0}\right)_{t} \overline{\left(\psi \cdot M^{0}\right)_{t}}\right)=\int_{0}^{t} d s \int_{\mathbb{R}^{d} \times \mathbb{R}^{d}} \Gamma_{0}(d x, d y) \phi(s, x) \overline{\psi(s, y)} .
$$

2.4. Noise on a hyperplane. Let $d \geq 2$ be an integer. For $x \in \mathbb{R}^{d} \equiv \mathbb{R}^{d-1} \times \mathbb{R}$, we write $x=\left(x_{1}, x_{2}\right)$, where $x_{1} \in \mathbb{R}^{d-1}$ and $x_{2} \in \mathbb{R}$. For $\varphi \in \mathcal{S}\left(\mathbb{R}^{d}\right)$, let $\mathcal{F}_{1} \varphi$ (resp. $\left.\mathcal{F}_{2} \varphi\right)$ denote the Fourier transform of $\varphi$ in the coordinates $x_{1}$ (resp. $x_{2}$ ):

$$
\mathcal{F}_{1} \varphi\left(\xi_{1}, x_{2}\right)=\int_{\mathbb{R}^{d-1}} d x_{1} \varphi\left(x_{1}, x_{2}\right) \chi_{\xi_{1}}\left(x_{1}\right)
$$

and

$$
\mathcal{F}_{2} \varphi\left(x_{1}, \xi_{2}\right)=\int_{\mathbb{R}} d x_{2} \varphi\left(x_{1}, x_{2}\right) \chi_{\xi_{2}}\left(x_{2}\right) .
$$

These Fourier transforms extend to $T \in \mathcal{S}^{\prime}\left(\mathbb{R}^{d}\right)$ by the relationship $\left\langle\mathcal{F}_{1} T, \varphi\right\rangle=$ $\left\langle T, \mathcal{F}_{1} \varphi\right\rangle$. Note that $\mathcal{F}=\mathcal{F}_{1} \circ \mathcal{F}_{2}=\mathcal{F}_{2} \circ \mathcal{F}_{1}$. 
We want to consider the situation of noise concentrated on the hyperplane $\mathbb{R}^{d-1} \times$ $\{0\}$ and spatially homogeneous within this hyperplane. Therefore, we shall assume that the measure $\Gamma_{0}$ is given by

$$
\Gamma_{0}(x, y)=\Gamma\left(x_{1}-y_{1}\right) \delta_{0}\left(x_{2}\right) \delta_{0}\left(y_{2}\right)
$$

or, in other words, for all $\phi, \psi \in \mathcal{S}\left(\mathbb{R}^{d}\right)$,

$$
\int_{\mathbb{R}^{d} \times \mathbb{R}^{d}} \Gamma_{0}(d x, d y) \phi(x) \psi(y)=\int_{\mathbb{R}^{d-1}} \Gamma\left(d z_{1}\right)\left(\phi(\cdot, 0) *_{1} \tilde{\psi}(\cdot, 0)\right)\left(z_{1}\right),
$$

where $*_{1}$ denotes the convolution product in $\mathbb{R}^{d-1}$ and $\tilde{\varphi}\left(x_{1}\right)=\overline{\varphi\left(-x_{1}\right)}$ for $x_{1} \in$ $\mathbb{R}^{d-1}$.

We assume that $\Gamma$ is a signed Borel measure on $\mathbb{R}^{d-1}$, which is non-negative definite on $\mathbb{R}^{d-1}$, that is,

$$
\int_{\mathbb{R}^{d-1}} \Gamma\left(d z_{1}\right)\left(\varphi *_{1} \tilde{\varphi}\right)\left(z_{1}\right) \geq 0, \quad \text { for all } \varphi \in \mathcal{S}\left(\mathbb{R}^{d-1}\right) .
$$

This implies that $\Gamma$ is symmetric (cf. 28, Chap. VII, Thm. XVII]) and by the Bochner-Schwartz theorem (see [28, Chap. VII, Thm. XVIII]), there is a nonnegative tempered measure $\mu$ such that $\Gamma=\mathcal{F} \mu$. Moreover, we assume that there exists a tempered non-negative Borel measure $\nu$ on $\mathbb{R}^{d-1}$ which is non-negative definite and which dominates $|\Gamma|$, that is,

$$
|\Gamma|(A) \leq \nu(A), \quad \text { for all } A \in \mathcal{B}_{b}\left(\mathbb{R}^{d-1}\right) .
$$

Example 2.1. (a) If $\Gamma$ is a non-negative, tempered and non-negative definite Borel measure on $\mathbb{R}^{d}$, then $\nu=\Gamma$ satisfies the required assumptions. This non-negativity assumption was taken as a basic assumption in [5, 27] (in the case of spatially homogeneous noise on $\mathbb{R}^{d}$ ) but we will only adopt it in our analysis of non-linear equations (see Section 5).

(b) For certain non-negative continuous functions $f$ on $] 0, \infty[$, covariances of the form $\Gamma\left(d x_{1}\right)=f\left(\left|x_{1}\right|\right) d x_{1}$ satisfy (2.10). Examples of such $f$ are

$$
\left.f(r)=r^{-\gamma}, \quad \text { where } \quad \gamma \in\right] 0, d-1[.
$$

(c) Let $\lambda$ be Lebesgue measure on $\mathbb{R}^{d-1}$. Suppose $\Gamma$ is a non-negative definite tempered Borel measure on $\mathbb{R}^{d}$ for which there exists $C>0$ such that

$$
\Gamma+C \lambda \text { is a non-negative measure on } \mathbb{R}^{d-1} .
$$

Then $\nu=\Gamma+2 C \lambda$ satisfies the required assumptions: $\nu$ is non-negative definite, being the convex combination of two non-negative definite measures, and

$$
|\Gamma|=|\Gamma+C \lambda-C \lambda| \leq|\Gamma+C \lambda|+|C \lambda|=\Gamma+2 C \lambda=\nu .
$$

Note that (2.11) was taken as a basic assumption in [25, 26] (in the case of spatially homogeneous noise on $\mathbb{R}^{d}$ ).

As in Section 2.3 we associate a Gaussian process $F^{0}$ with $\Gamma_{0}$. On the other hand, we can consider the centered Gaussian process $F=\left\{F_{t}(\varphi), t \in \mathbb{R}_{+}, \varphi \in\right.$ $\left.\mathcal{S}\left(\mathbb{R}^{d-1}\right)\right\}$ whose covariance is given for all $t, s \in \mathbb{R}_{+}$and $\varphi, \psi \in \mathcal{S}\left(\mathbb{R}^{d-1}\right)$ by

$$
\mathbb{E}\left(F_{t}(\varphi) \overline{F_{s}(\psi)}\right)=(t \wedge s) \int_{\mathbb{R}^{d-1}} \Gamma\left(d z_{1}\right)\left(\varphi *_{1} \tilde{\psi}\right)\left(z_{1}\right)
$$


By the Kolmogorov extension theorem (see [23, Prop. 3.4]), this process is well defined on some probability space $(\Omega, \mathcal{G}, \mathbb{P})$, and (2.9) implies that

$$
F_{t}^{0}(\phi) \stackrel{d}{=} F_{t}(\phi(\cdot, 0)), \quad \text { for all } t \in \mathbb{R}_{+}, \phi \in \mathcal{S}\left(\mathbb{R}^{d}\right),
$$

where $\stackrel{d}{=}$ stands for equality in distribution. Setting $u_{0}=v_{0}=0$, since we are mainly interested in studying how the regularity of the solution depends on the regularity of the noise, equation (2.1) may then be formally rewritten as

$$
\left\{\begin{array}{rl}
\frac{\partial^{2} u}{\partial t^{2}}(t, x)+2 a \frac{\partial u}{\partial t}(t, x)+b & u(t, x)-\Delta u(t, x) \\
& =\dot{F}\left(t, x_{1}\right) \delta_{0}\left(x_{2}\right), \quad(t, x) \in \mathbb{R}_{+} \times \mathbb{R}^{d},
\end{array}\right.
$$

2.5. Section 2.3 revisited. We particularize the definitions of Section 2.3 to the noise $F$ on a hyperplane defined in Section 2.4. Let $\left\{\mathcal{G}_{t}\right\}$ denote the natural augmented filtration of the noise $F, \mathcal{E}$ the space of elementary integrands (which are now functions on $\mathbb{R}_{+} \times \mathbb{R}^{d-1} \times \Omega$ ) and $\mathcal{P}$ the predictable $\sigma$-field; the noise $F$ extends naturally to a worthy martingale measure $M$ and we can define a corresponding stochastic integral $(\phi \cdot M)_{t}$ for integrands belonging to the set $H_{t,+}$ of predictable $\phi:[0, t] \times \mathbb{R}^{d-1} \times \Omega \rightarrow \mathbb{C}$ such that

$$
\|\phi\|_{t,+}^{2}=\mathbb{E}\left(\int_{0}^{t} d s \int_{\mathbb{R}^{d-1}} \nu\left(d z_{1}\right)\left(|\phi(s, \cdot)| *_{1}|\tilde{\phi}(s, \cdot)|\right)\left(z_{1}\right)\right)<\infty,
$$

using the isometry

$$
\mathbb{E}\left((\phi \cdot M)_{t} \overline{(\psi \cdot M)_{t}}\right)=\langle\phi, \psi\rangle_{t}=\mathbb{E}\left(\int_{0}^{t} d s \int_{\mathbb{R}^{d-1}} \Gamma\left(d z_{1}\right)\left(\phi(s, \cdot) *_{1} \tilde{\psi}(s, \cdot)\right)\left(z_{1}\right)\right) .
$$

Let $\|\cdot\|_{t}$ denote the semi-norm induced by the semi-inner product $\langle\cdot, \cdot\rangle_{t}$.

We will adopt the following notation for the stochastic integral of a predictable integrand $\phi:[0, t] \times \mathbb{R}^{d} \times \mathbb{R} \times \Omega \rightarrow \mathbb{C}$ restricted to the hyperplane $x_{2}=0$ :

$$
(\phi(\cdot, \cdot, 0) \cdot M)_{t}=\int_{[0, t] \times \mathbb{R}^{d-1}} M\left(d s, d x_{1}\right) \phi\left(s, x_{1}, 0\right) .
$$

2.6. Extension of the stochastic integral. The first technical step towards the study of the regularity of the solution of (2.12) consists in extending the stochastic integral to distribution-valued integrands, since the processes that will appear in the following will be expressed as stochastic integrals of such integrands.

Following [5], let $Z=\left\{Z\left(t, x_{1}\right),\left(t, x_{1}\right) \in \mathbb{R}_{+} \times \mathbb{R}^{d-1}\right\}$ be a real-valued predictable process such that for all $T>0$,

$$
\sup _{\left(t, x_{1}\right) \in[0, T] \times \mathbb{R}^{d-1}} \mathbb{E}\left(Z\left(t, x_{1}\right)^{2}\right)<\infty .
$$

By [30, Chap. 2], $M^{Z}=\left\{(Z \cdot M)_{t}(B), \mathcal{G}_{t}, t \in \mathbb{R}_{+}, B \in \mathcal{B}_{b}\left(\mathbb{R}^{d-1}\right)\right\}$ defines a worthy martingale measure with covariation measure

$$
Q^{Z}([0, t] \times A \times B)=\mathbb{E}\left(\int_{0}^{t} d s \int_{\mathbb{R}^{d-1}} \Gamma\left(d z_{1}\right)\left(\left(Z(s, \cdot) 1_{A}\right) *_{1}\left(\tilde{Z}(s, \cdot) \tilde{1}_{B}\right)\right)\left(z_{1}\right)\right)
$$


and dominating measure

$$
K^{Z}([0, t] \times A \times B)=\mathbb{E}\left(\int_{0}^{t} d s \int_{\mathbb{R}^{d-1}} \nu\left(d z_{1}\right)\left(\left(|Z(s, \cdot)| 1_{A}\right) *_{1}\left(|\tilde{Z}(s, \cdot)| \tilde{1}_{B}\right)\right)\left(z_{1}\right)\right) .
$$

This implies that we can define the stochastic integral $\left(\phi \cdot M^{Z}\right)_{t}$ of a Borel-measurable function $\phi:[0, t] \times \mathbb{R}^{d-1} \rightarrow \mathbb{C}$ such that

$$
\|\phi\|_{t,+, Z}^{2}=\mathbb{E}\left(\int_{0}^{t} d s \int_{\mathbb{R}^{d-1}} \nu\left(d z_{1}\right)\left(|\phi(s, \cdot) Z(s, \cdot)| *_{1}|\tilde{\phi}(s, \cdot) \tilde{Z}(s, \cdot)|\right)\left(z_{1}\right)\right)<\infty,
$$

and let us denote by $H_{t,+, Z}$ the space of such (deterministic) integrands. Note that if $\phi \in H_{t,+}$ and $\phi$ is deterministic, then

$$
\|\phi\|_{t,+, Z}^{2} \leq \sup _{\left(s, x_{1}\right) \in[0, t] \times \mathbb{R}^{d-1}} \mathbb{E}\left(Z\left(s, x_{1}\right)^{2}\right)\|\phi\|_{t,+}^{2}<\infty,
$$

so $\phi \in H_{t,+, Z}$. Moreover, the following isometry property holds:

$$
\mathbb{E}\left(\left(\phi \cdot M^{Z}\right)_{t} \overline{\left(\psi \cdot M^{Z}\right)_{t}}\right)=\langle\phi, \psi\rangle_{t, Z}
$$

where

$$
\langle\phi, \psi\rangle_{t, Z}=\mathbb{E}\left(\int_{0}^{t} d s \int_{\mathbb{R}^{d-1}} \Gamma\left(d z_{1}\right)\left((\phi(s, \cdot) Z(s, \cdot)) *_{1}(\tilde{\psi}(s, \cdot) \tilde{Z}(s, \cdot))\right)\left(z_{1}\right)\right) .
$$

Let us also denote by $\|\cdot\|_{t, Z}$ the semi-norm induced by the semi-inner product $\langle\cdot, \cdot\rangle_{t, Z}$

We can now proceed to the extension of the stochastic integral. If we assume that $Z$ satisfies

$\mathbb{E}\left(Z\left(t, x_{1}\right) Z\left(t, y_{1}\right)\right)=\mathbb{E}\left(Z(t, 0) Z\left(t, x_{1}-y_{1}\right)\right), \quad$ for all $t \in \mathbb{R}_{+}, x_{1}, y_{1} \in \mathbb{R}^{d-1}$,

then the function $\gamma: \mathbb{R}_{+} \times \mathbb{R}^{d-1} \rightarrow \mathbb{R}$ defined by

$$
\gamma\left(t, z_{1}\right)=\mathbb{E}\left(Z(t, 0) Z\left(t, z_{1}\right)\right), \quad\left(t, z_{1}\right) \in \mathbb{R}_{+} \times \mathbb{R}^{d-1},
$$

is symmetric and non-negative definite in $z_{1}$ and for deterministic $\phi, \psi \in H_{t,+, Z}$, (2.16) can be rewritten as

$$
\langle\phi, \psi\rangle_{t, Z}=\int_{0}^{t} d s \int_{\mathbb{R}^{d-1}} \Gamma\left(d z_{1}\right) \gamma\left(s, z_{1}\right)\left(\phi(s, \cdot) *_{1} \tilde{\psi}(s, \cdot)\right)\left(z_{1}\right) .
$$

Notice that for $s \in[0, t]$, the measure $\Gamma_{s}^{Z}$ defined by

$$
\Gamma_{s}^{Z}\left(d z_{1}\right)=\Gamma\left(d z_{1}\right) \gamma\left(s, z_{1}\right)
$$

is a non-negative definite measure on $\mathbb{R}^{d-1}$, since this property is conserved by multiplication of non-negative definite functions/measures.

By the Bochner-Schwartz theorem (see [28, Chap. VII, Thm. XVIII]), there exists a non-negative tempered Borel measure $\mu_{s}^{Z}$ on $\mathbb{R}^{d-1}$ such that $\Gamma_{s}^{Z}=\mathcal{F}_{1} \mu_{s}^{Z}$. Moreover, $\mu_{s}^{Z}$ is symmetric on $\mathbb{R}^{d-1}$, since $\Gamma_{s}^{Z}$ is real-valued. Let us now consider 
the following subspace of $H_{t,+}$ :

$$
\begin{aligned}
H_{t, 0}=\left\{\phi:[0, t] \times \mathbb{R}^{d-1} \rightarrow \mathbb{C}\right. \text { Borel-measurable such that } \\
\left.\qquad \phi \|_{t,+}<\infty \text { and } \phi(s, \cdot) \in \mathcal{S}\left(\mathbb{R}^{d-1}\right), \quad \text { for all } s \in[0, t]\right\} .
\end{aligned}
$$

If $\phi, \psi \in H_{t, 0}$, then $\mathcal{F}_{1} \phi, \mathcal{F}_{1} \psi$ are Borel-measurable functions and we obtain the following expression for $\|\phi\|_{t, Z}$, using basic properties of the Fourier transform:

$$
\|\phi\|_{t, Z}^{2}=\int_{0}^{t} d s \int_{\mathbb{R}^{d-1}} \mu_{s}^{Z}\left(d \xi_{1}\right)\left|\mathcal{F}_{1} \phi\left(s, \xi_{1}\right)\right|^{2} .
$$

Note that this formula defines $\|\phi\|_{t, Z}$ even for $\phi:[0, t] \rightarrow O_{C}^{\prime}\left(\mathbb{R}^{d-1}\right)$ such that $\left(s, \xi_{1}\right) \mapsto \mathcal{F}_{1} \phi\left(s, \xi_{1}\right)$ is Borel-measurable. We now define a larger space, which contains (deterministic) distribution-valued integrands:

$$
\begin{aligned}
H_{t, Z}=\{\phi:[0, t] & \rightarrow O_{C}^{\prime}\left(\mathbb{R}^{d-1}\right):\left(s, \xi_{1}\right) \mapsto \mathcal{F}_{1} \phi\left(s, \xi_{1}\right) \text { is Borel-measurable, } \\
& \left.\|\phi\|_{t, Z}<\infty \text { and } \exists\left(\phi_{n}\right) \subset H_{t, 0} \text { such that }\left\|\phi-\phi_{n}\right\|_{t, Z} \underset{n \rightarrow \infty}{\rightarrow} 0\right\}
\end{aligned}
$$

(note that as in [5], it is not known if the set $H_{t, Z}$ would remain the same if the condition of existence of the approximating sequence $\left(\phi_{n}\right)$ were removed). The stochastic integral $\left(\phi \cdot M^{Z}\right)_{t}$ then extends by isometry to elements of $H_{t, Z}$. We continue to use the notation (2.14) for stochastic integrals even in the case where $\phi\left(s, \cdot, x_{2}\right)$ is a distribution in $x_{1}$ for each $x_{2} \in \mathbb{R}$.

Note that for the linear equation, we will only need the definition of the stochastic integral when $Z \equiv 1$, in which case we denote the space of integrands by $H_{t}$ and the isometry property (2.13) becomes

$$
\mathbb{E}\left((\phi \cdot M)_{t} \overline{(\psi \cdot M)_{t}}\right)=\langle\phi, \psi\rangle_{t}=\int_{0}^{t} d s \int_{\mathbb{R}^{d-1}} \mu\left(d \xi_{1}\right) \mathcal{F}_{1} \phi\left(s, \xi_{1}\right) \overline{\mathcal{F}_{1} \psi\left(s, \xi_{1}\right)}
$$

Notice that since $\phi$ is deterministic, the process $(\phi \cdot M)=\left\{(\phi \cdot M)_{t}, t \in \mathbb{R}_{+}\right\}$is a Gaussian process.

The following theorems will also be useful (cf. [5, Thms. 2 and 3] and [6] for proofs, noting that Theorem 2.3 is a slight variation on the result of [6]). Before stating them, let us denote by $O_{C}^{\prime}\left(\mathbb{R}^{d-1}\right)_{+}$the space of non-negative distributions with rapid decrease on $\mathbb{R}^{d-1}$.

Theorem 2.2. Let $Z$ be a process satisfying (2.15) and (2.17). If $\Gamma$ is a nonnegative measure on $\mathbb{R}^{d-1}, \phi:[0, t] \rightarrow O_{C}^{\prime}\left(\mathbb{R}^{d-1}\right)_{+}$is such that $\mathcal{F}_{1} \phi$ is a Borelmeasurable function and $\|\phi\|_{t}<\infty$, then $\phi \in H_{t, Z}$ and

$$
\begin{aligned}
\mathbb{E}\left(\left|\left(\phi \cdot M^{Z}\right)_{t}\right|^{2}\right) & =\int_{0}^{t} d s \int_{\mathbb{R}^{d-1}} \mu_{s}^{Z}\left(d \xi_{1}\right)\left|\mathcal{F}_{1} \phi\left(s, \xi_{1}\right)\right|^{2} \\
& \leq \int_{0}^{t} d s \sup _{x_{1} \in \mathbb{R}^{d-1}} \mathbb{E}\left(Z\left(s, x_{1}\right)^{2}\right) \int_{\mathbb{R}^{d-1}} \mu\left(d \xi_{1}\right)\left|\mathcal{F}_{1} \phi\left(s, \xi_{1}\right)\right|^{2} .
\end{aligned}
$$

Theorem 2.3. If $\phi:[0, t] \rightarrow O_{C}^{\prime}\left(\mathbb{R}^{d-1}\right)$ is such that $\mathcal{F}_{1} \phi$ is a Borel-measurable function, $\|\phi\|_{t}<\infty$ and

$$
\lim _{h \downarrow 0} \int_{0}^{t} d s \int_{\mathbb{R}^{d-1}} \mu\left(d \xi_{1}\right) \sup _{s<r<s+h}\left|\mathcal{F}_{1} \phi\left(r, \xi_{1}\right)-\mathcal{F}_{1} \phi\left(s, \xi_{1}\right)\right|^{2}=0,
$$

then $\phi \in H_{t}$. 


\section{The Green KeRnel AND ITS PROPERTIES}

Let $G$ be the solution of

$$
\frac{\partial^{2} G}{\partial t^{2}}+2 a \frac{\partial G}{\partial t}+b G-\Delta G=0, \quad G(0)=0, \quad \frac{\partial G}{\partial t}(0)=\delta_{0}
$$

$G$ is termed the Green kernel of equation (2.1). Note that in the following, the dependence on $a$ or $b$ of the Green kernel or other objects will be omitted in order to simplify the notation.

3.1. Fourier transform of the Green kernel. As for instance in [5, Example 7], one immediately checks that the Fourier transform of $G$ in $x$ satisfies

$$
\left\{\begin{array}{l}
\frac{\partial^{2} \mathcal{F} G}{\partial t^{2}}(t, \xi)+2 a \frac{\partial \mathcal{F} G}{\partial t}(t, \xi)+\left(b+|\xi|^{2}\right) \mathcal{F} G(t, \xi)=0, \quad t \in \mathbb{R}, \quad \xi \in \mathbb{R}^{d}, \\
\mathcal{F} G(0, \xi)=0, \quad \frac{\partial}{\partial t} \mathcal{F} G(0, \xi)=1, \quad \xi \in \mathbb{R}^{d} .
\end{array}\right.
$$

For $\xi$ fixed, this is an ordinary differential equation in $t$, whose solution yields the following expression for $\mathcal{F} G$, which is valid for all dimensions $d$ :

$$
\mathcal{F} G(t, \xi)= \begin{cases}e^{-a t} \frac{\sin \left(t \sqrt{|\xi|^{2}+b-a^{2}}\right)}{\sqrt{|\xi|^{2}+b-a^{2}}}, & \text { if }|\xi|^{2}>a^{2}-b, \\ e^{-a t} t, & \text { if } a^{2}-b \geq 0 \text { and }|\xi|^{2}=a^{2}-b, \\ e^{-a t} \frac{\sinh \left(t \sqrt{a^{2}-b-|\xi|^{2}}\right)}{\sqrt{a^{2}-b-|\xi|^{2}}}, & \text { if } a^{2}-b>0 \text { and }|\xi|^{2}<a^{2}-b .\end{cases}
$$

Note that the first of these three expressions contains the other two. From these, we observe that $\mathcal{F} G(t, \cdot)$ is an even real-valued function on $\mathbb{R}^{d}$. Moreover, it is a well-known fact (see for instance [16, Thm 12.5.1]) that for fixed $t \in \mathbb{R}_{+}, G(t, \cdot)$ is a distribution on $\mathbb{R}^{d}$ compactly supported in $B_{d}(0, t)$ (the ball in $\mathbb{R}^{d}$ centered at 0 with radius $t$ ), so $G(t, \cdot) \in O_{C}^{\prime}\left(\mathbb{R}^{d}\right)$ and $\mathcal{F} G(t, \cdot) \in O_{M}\left(\mathbb{R}^{d}\right)$ by (2.3).

Let us also define $H=\frac{\partial G}{\partial t}+2 a G$. Then

$$
\frac{\partial^{2} H}{\partial t^{2}}+2 a \frac{\partial H}{\partial t}+b H-\Delta H=0, \quad H(0)=\delta_{0}, \quad \frac{\partial H}{\partial t}(0)=0 .
$$

Indeed, the first two equalities follow directly from the definition of $H$ and (3.1), and the third inequality is obtained by computing $\mathcal{F} H(t, \xi)$ from (3.3).

3.2. Fourier transform in $x_{1}$ of the Green kernel. We first need to establish some properties of the restriction (or trace) of the solution $G$ of equation (3.1) to the hyperplane $\mathbb{R}^{d-1} \times\left\{x_{2}\right\}$ and of its Fourier transform in the first $d-1$ coordinates of $x$ (cf. Section 2.4). For $\left(t, \xi_{1}, x_{2}\right) \in \mathbb{R}_{+} \times \mathbb{R}^{d-1} \times \mathbb{R}$, set

$$
L_{1}\left(t, \xi_{1}, x_{2}\right)=\mathcal{F}_{2}^{-1}\left(\mathcal{F} G\left(t, \xi_{1}, \cdot\right)\right)\left(x_{2}\right)=\frac{1}{2 \pi} \int_{\mathbb{R}} d \xi_{2} \mathcal{F} G\left(t, \xi_{1}, \xi_{2}\right) \chi_{-\xi_{2}}\left(x_{2}\right),
$$


by (2.2). Using (3.3) and [24, formulas I.5.83 and I.7.61], we see that

$$
\begin{aligned}
& L_{1}\left(t, \xi_{1}, x_{2}\right) \\
& \quad=\left\{\begin{array}{lll}
\frac{e^{-a t}}{2} J_{0}\left(\sqrt{\left(\left|\xi_{1}\right|^{2}+b-a^{2}\right)\left(t^{2}-x_{2}^{2}\right)}\right) & 1_{\left\{\left|x_{2}\right| \leq t\right\}}, & \text { if } \quad\left|\xi_{1}\right|^{2} \geq a^{2}-b, \\
\frac{e^{-a t}}{2} I_{0}\left(\sqrt{\left(a^{2}-b-\left|\xi_{1}\right|^{2}\right)\left(t^{2}-x_{2}^{2}\right)}\right) & 1_{\left\{\left|x_{2}\right| \leq t\right\}}, & \text { if } \quad a^{2}-b>0 \\
& \text { and }\left|\xi_{1}\right|^{2}<a^{2}-b,
\end{array}\right.
\end{aligned}
$$

where $J_{0}$ and $I_{0}$ are respectively the zero order regular and modified Bessel functions of the first kind (see Appendix C). In particular, $L_{1}$ is a real-valued function, which is bounded on $[0, T] \times \mathbb{R}^{d-1} \times \mathbb{R}$ for all $T>0$ and symmetric in $\xi_{1}$. Further, the element of $S^{\prime}\left(\mathbb{R}^{d}\right)$ associated with the function $\left(\xi_{1}, x_{2}\right) \mapsto L_{1}\left(t, \xi_{1}, x_{2}\right)$ is $\mathcal{F}_{1} G(t)(\cdot)$. Indeed, for $\varphi \in \mathcal{S}\left(\mathbb{R}^{d}\right)$,

$$
\begin{array}{rl}
\int_{\mathbb{R}^{d-1}} & d \xi_{1} \int_{\mathbb{R}} d x_{2} L_{1}\left(t, \xi_{1}, x_{2}\right) \varphi\left(\xi_{1}, x_{2}\right) \\
= & \int_{\mathbb{R}^{d-1}} d \xi_{1} \int_{\mathbb{R}} d x_{2} \int_{\mathbb{R}} d \xi_{2} \mathcal{F} G\left(t, \xi_{1}, \xi_{2}\right) \chi_{-\xi_{2}}\left(x_{2}\right) \varphi\left(\xi_{1}, x_{2}\right) \\
= & \int_{\mathbb{R}^{d-1}} d \xi_{1} \int_{\mathbb{R}} d \xi_{2} \mathcal{F} G\left(t, \xi_{1}, \xi_{2}\right) \mathcal{F}_{2}^{-1} \varphi\left(\xi_{1}, x_{2}\right) \\
= & \left\langle\mathcal{F}_{1} G(t), \varphi\right\rangle .
\end{array}
$$

Proposition 3.1. For $\left(t, x_{2}\right) \in \mathbb{R}_{+} \times \mathbb{R}$, define $G_{1}\left(t, \cdot, x_{2}\right)=\mathcal{F}_{1}^{-1} L_{1}\left(t, \cdot, x_{2}\right)$, or, equivalently, $\mathcal{F}_{1} G_{1}\left(t, \cdot, x_{2}\right)=L_{1}\left(t, \cdot, x_{2}\right)$. Then $G_{1}\left(t, \cdot, x_{2}\right) \in O_{C}^{\prime}\left(\mathbb{R}^{d-1}\right)$ and for $\varphi \in \mathcal{S}\left(\mathbb{R}^{d}\right)$

$$
\langle G(t), \varphi\rangle=\int_{\mathbb{R}} d x_{2}\left\langle G_{1}\left(t, \cdot, x_{2}\right), \varphi\left(\cdot, x_{2}\right)\right\rangle .
$$

Proof. Using the definition of $G_{1}$ and (3.6), we see that the right-hand side of (3.7) is equal to

$$
\begin{aligned}
\int_{\mathbb{R}} d x_{2} \int_{\mathbb{R}^{d-1}} d \xi_{1} L_{1}\left(t, \xi_{1}, x_{2}\right) \mathcal{F}_{1}^{-1} \varphi\left(\xi_{1}, x_{2}\right) & =\left\langle\mathcal{F}_{1} G(t), \mathcal{F}_{1}^{-1} \varphi\right\rangle \\
& =\langle G(t), \varphi\rangle .
\end{aligned}
$$

This establishes (3.7), and so $G_{1}\left(t, \cdot, x_{2}\right)$ is a distribution on $\mathbb{R}^{d-1}$ with compact support in $B_{d-1}(0, t)$. Therefore, $G\left(t, \cdot, x_{2}\right) \in O_{C}^{\prime}\left(\mathbb{R}^{d-1}\right)$, and this completes the proof.

According to Proposition 3.1 for fixed $t \in \mathbb{R}_{+}$and $x_{2} \in \mathbb{R}, G_{1}\left(t, \cdot x_{2}\right)$ can be interpreted as the restriction (or trace) of $G(t, \cdot)$ to the hyperplane $\mathbb{R}^{d-1} \times\left\{x_{2}\right\}$.

Example 3.2. Let $B_{d}(x, r)$ denote the open ball in $\mathbb{R}^{d}$ centered at $x$ with radius $r$. In the case $a=b=0$, (3.1) is the wave equation, and it is well known 15, Thms. 
5.15 and 5.17] that the Green kernel is given, for $d$ even, by

$$
\begin{aligned}
&\langle G(s), \varphi\rangle=C_{d}\left(\frac{1}{s} \frac{\partial}{\partial s}\right)^{N(d)} {\left[s^{d-1} \int_{B_{d}(0,1)} \frac{d y}{\sqrt{1-|y|^{2}}} \varphi(s y)\right] } \\
&=C_{d}\left(\frac{1}{s} \frac{\partial}{\partial s}\right)^{N(d)}\left[s^{d-1} \int_{-1}^{1} d y_{2} \int_{B_{d-1}\left(0, \sqrt{1-y_{2}^{2}}\right)} d y_{1}\right. \\
&\left.\cdot \frac{1}{\sqrt{1-y_{2}^{2}-\left|y_{1}\right|^{2}}} \varphi\left(s y_{1}, s y_{2}\right)\right],
\end{aligned}
$$

while for $d$ odd, it is given by

$$
\begin{array}{r}
\langle G(s), \varphi\rangle=C_{d}\left(\frac{1}{s} \frac{\partial}{\partial s}\right)^{N(d)}\left[s^{d-2} \int_{\partial B_{d}(0,1)} \sigma^{(d)}(d y) \varphi(s y)\right] \\
=C_{d}\left(\frac{1}{s} \frac{\partial}{\partial s}\right)^{N(d)}\left[s^{d-2} \int_{-1}^{1} d y_{2} \int_{\partial B_{d-1}\left(0, \sqrt{1-y_{2}^{2}}\right)}\right. \\
\left.\cdot \sigma^{(d-1)}\left(d y_{1}\right) \varphi\left(s y_{1}, s y_{2}\right)\right],
\end{array}
$$

where $N(d)=[(d-2) / 2](\operatorname{resp} .[(d-3) / 2])$ when $d$ is even (resp. odd), $\sigma^{(d)}$ is $(d-1)$ dimensional Hausdorff measure, and $C_{d}$ is a constant. Therefore, for $\left|x_{2}\right|<1$ and $d$ even,

$$
\begin{aligned}
& \left\langle G_{1}\left(s, \cdot s x_{2}\right), \varphi\left(\cdot, s x_{2}\right)\right\rangle \\
& \quad=\frac{C_{d}}{s}\left(\frac{1}{s} \frac{\partial}{\partial s}\right)^{N(d)}\left[s^{d-1} \int_{B_{d-1}\left(0, \sqrt{1-x_{2}^{2}}\right)} d y_{1} \frac{1}{\sqrt{1-x_{2}^{2}-\left|y_{1}\right|^{2}}} \varphi\left(s y_{1}, s x_{2}\right)\right],
\end{aligned}
$$

and for $d$ odd,

$$
\begin{aligned}
& \left\langle G_{1}\left(s, \cdot, s x_{2}\right), \varphi\left(\cdot, s x_{2}\right)\right\rangle \\
& \quad=\frac{C_{d}}{s}\left(\frac{1}{s} \frac{\partial}{\partial s}\right)^{N(d)}\left[s^{d-2} \int_{\partial B_{d-1}\left(0, \sqrt{1-x_{2}^{2}}\right)} \sigma^{(d-1)}\left(d y_{1}\right) \varphi\left(s y_{1}, s x_{2}\right)\right] .
\end{aligned}
$$

Our main results will be based on the estimates in the next three lemmas.

Lemma 3.3. For all $t>0$, there exists $C(t)>0$ such that for all $s \in[0, t]$, $\xi_{1} \in \mathbb{R}^{d-1}$ and $x_{2} \in \mathbb{R}$,

$$
\mathcal{F}_{1} G_{1}\left(s, \xi_{1}, x_{2}\right)^{2} \leq \frac{C(t)}{\sqrt{1+\left|\xi_{1}\right|^{2}}} \frac{1}{\sqrt{s^{2}-x_{2}^{2}}} 1_{\left\{\left|x_{2}\right|<s\right\}} .
$$

Proof. For fixed $t>0$, for $\xi_{1}$ bounded and for all $s<t$ and $x_{2} \in \mathbb{R}$ with $\left|x_{2}\right|<s$, the right-hand side of (3.8) is bounded below by a positive constant and the lefthand side is bounded above. Therefore, it suffices to prove (3.8) for $\left|\xi_{1}\right|$ sufficiently large, all $s<t$ and $x_{2} \in \mathbb{R}$ with $\left|x_{2}\right|<s$. In this case,

$$
\begin{aligned}
\mathcal{F}_{1} G_{1}\left(s, \xi_{1}, x_{2}\right)^{2} & =\frac{e^{-2 a s}}{4} J_{0}^{2}\left(\sqrt{\left(\left|\xi_{1}\right|^{2}+b-a^{2}\right)\left(s^{2}-x_{2}^{2}\right)}\right) 1_{\left\{\left|x_{2}\right|<s\right\}} \\
& \leq \frac{e^{2 a^{-} t}}{4} \frac{C}{\sqrt{\left|\xi_{1}\right|^{2}+b-a^{2}}} \frac{1}{\sqrt{s^{2}-x_{2}^{2}}} 1_{\left\{\left|x_{2}\right|<s\right\}}
\end{aligned}
$$


by (C.2), where $a^{-}=\max (-a, 0)$. The conclusion is now clear, since

$$
2 \sqrt{\left|\xi_{1}\right|^{2}+b-a^{2}} \geq \sqrt{1+\left|\xi_{1}\right|^{2}}
$$

for $\left|\xi_{1}\right|$ sufficiently large.

Lemma 3.4. (a) For all $t>0$, there exists $C(t)>0$ such that for all $\xi_{1} \in \mathbb{R}^{d-1}$ and $x_{2} \in \mathbb{R}^{*}:=\mathbb{R} \backslash\{0\}$,

$$
\int_{0}^{t} d s \mathcal{F}_{1} G_{1}\left(s, \xi_{1}, x_{2}\right)^{2} \leq \frac{C(t)}{\sqrt{1+\left|\xi_{1}\right|^{2}}} \operatorname{arccosh}\left(\frac{t}{\left|x_{2}\right|}\right) 1_{\left\{\left|x_{2}\right|<t\right\}} .
$$

(b) For all $t>0$, there exists $C(t)>0$ such that for all $\xi_{1} \in \mathbb{R}^{d-1}$ and $x_{2} \in \mathbb{R}$,

$$
\int_{0}^{t} d s \mathcal{F}_{1} G_{1}\left(s, \xi_{1}, x_{2}\right)^{2} \leq C(t) \frac{1+\ln \left(1+\left|\xi_{1}\right|^{2}\right)}{\sqrt{1+\left|\xi_{1}\right|^{2}}} .
$$

Proof. (a) We obtain (3.9) by integrating in $s$ both sides of (3.8).

(b) For fixed $t>0$, the left-hand side of (3.10) is a continuous function of $\xi_{1}$, which is therefore bounded above for all $x_{2}$ and $\left|\xi_{1}\right|$ bounded. On the other hand, the right-hand side is bounded below by a positive constant for $\left|\xi_{1}\right|$ bounded. Therefore, it suffices to check (3.10) for $\left|\xi_{1}\right|$ sufficiently large. In this case,

$$
\begin{aligned}
\int_{0}^{t} d s & \mathcal{F}_{1} G_{1}\left(s, \xi_{1}, x_{2}\right)^{2} \\
& =\int_{0}^{t} d s \frac{e^{-2 a s}}{4} J_{0}^{2}\left(\sqrt{\left(\left|\xi_{1}\right|^{2}+b-a^{2}\right)\left(s^{2}-x_{2}^{2}\right)}\right) 1_{\left\{\left|x_{2}\right|<s\right\}} \\
& \leq \frac{C e^{2 a^{-} t}}{4} \int_{\left|x_{2}\right|}^{t} d s \frac{1}{\sqrt{1+\left(\left|\xi_{1}\right|^{2}+b-a^{2}\right)\left(s^{2}-x_{2}^{2}\right)}} 1_{\left\{\left|x_{2}\right|<t\right\}},
\end{aligned}
$$

by (C.2). Using the formula

$$
\int \frac{1}{\sqrt{\alpha+s^{2}}} d s=\ln \left(s+\sqrt{\alpha+s^{2}}\right)
$$

we find that

$$
\begin{aligned}
& \int_{0}^{t} d s \mathcal{F}_{1} G_{1}\left(s, \xi_{1}, x_{2}\right)^{2} \\
& \quad \leq \frac{C e^{2 a^{-} t}}{\sqrt{\left|\xi_{1}\right|^{2}+b-a^{2}}} \ln \left(\frac{t+\sqrt{\frac{1}{\left|\xi_{1}\right|^{2}+b-a^{2}}+t^{2}-x_{2}^{2}}}{\left|x_{2}\right|+\frac{1}{\sqrt{\left|\xi_{1}\right|^{2}+b-a^{2}}}}\right) 1_{\left\{\left|x_{2}\right|<t\right\}} .
\end{aligned}
$$

This last expression is maximum when $x_{2}=0$, in which case it is bounded above for $\left|\xi_{1}\right|$ large by the right-hand side of (3.10).

Lemma 3.5. (a) For all $t>0$ and $x_{2} \in \mathbb{R}$ such that $\left|x_{2}\right|<t$, there exists $C\left(t, x_{2}\right)>$ 0 such that for all $\xi_{1} \in \mathbb{R}^{d-1}$,

$$
\int_{0}^{t} d s \mathcal{F}_{1} G_{1}\left(s, \xi_{1}, x_{2}\right)^{2} \geq \frac{C\left(t, x_{2}\right)}{\sqrt{1+\left|\xi_{1}\right|^{2}}} .
$$

(b) For all $t>0$, there exist $C(t)>0$ such that for all $\xi_{1} \in \mathbb{R}^{d-1}$,

$$
\int_{0}^{t} d s \mathcal{F}_{1} G_{1}\left(s, \xi_{1}, 0\right)^{2} \geq C(t) \frac{\ln \left(1+\left|\xi_{1}\right|^{2}\right)}{\sqrt{1+\left|\xi_{1}\right|^{2}}}
$$


Proof. (a) For fixed $t>0$ and $x_{2} \in \mathbb{R}$, the left-hand side of (3.12) is a positive and continuous function of $\left|\xi_{1}\right|$, which is therefore bounded below by a positive constant for $\left|\xi_{1}\right|$ bounded. On the other hand, the right-hand side is bounded above. Therefore, it suffices to prove (3.12) for $\left|\xi_{1}\right|$ sufficiently large. In this case,

$$
\begin{aligned}
\int_{0}^{t} d s & \mathcal{F}_{1} G_{1}\left(s, \xi_{1}, x_{2}\right)^{2} \\
= & \int_{0}^{t} d s \frac{e^{-2 a s}}{4} J_{0}^{2}\left(\sqrt{\left(\left|\xi_{1}\right|^{2}+b-a^{2}\right)\left(s^{2}-x_{2}^{2}\right)}\right) 1_{\left\{\left|x_{2}\right|<s\right\}} \\
& \geq \frac{e^{-2 a^{+} t}}{4} \int_{\left|x_{2}\right|}^{t} d s J_{0}^{2}\left(\sqrt{\left(\left|\xi_{1}\right|^{2}+b-a^{2}\right)\left(s^{2}-x_{2}^{2}\right)}\right),
\end{aligned}
$$

where $a^{+}=\max (a, 0)$. Use the change of variables $r=\sqrt{\left(\left|\xi_{1}\right|^{2}+b-a^{2}\right)\left(s^{2}-x_{2}^{2}\right)}$, so that

$$
d s=\frac{r d r}{s\left(\left|\xi_{1}\right|^{2}+b-a^{2}\right)} \geq \frac{r d r}{t\left(\left|\xi_{1}\right|^{2}+b-a^{2}\right)},
$$

and set $R=\sqrt{\left(\left|\xi_{1}\right|^{2}+b-a^{2}\right)\left(t^{2}-x_{2}^{2}\right)}$ to see that

$$
\int_{0}^{t} d s \mathcal{F}_{1} G_{1}\left(s, \xi_{1}, x_{2}\right)^{2} \geq \frac{e^{-2 a^{+} t}}{4 t} \frac{1}{\left|\xi_{1}\right|^{2}+b-a^{2}} \int_{0}^{R} d r r J_{0}^{2}(r) .
$$

Since $R \geq 1$ for large $\left|\xi_{1}\right|$, we obtain using Lemma C.1 that for such $\xi_{1}$,

$$
\begin{aligned}
\int_{0}^{t} d s \mathcal{F}_{1} G_{1}\left(s, \xi_{1}, x_{2}\right)^{2} & \geq \frac{C e^{-2 a^{+} t}}{4 t} \frac{\sqrt{\left(\left|\xi_{1}\right|^{2}+b-a^{2}\right)\left(t^{2}-x_{2}^{2}\right)}}{\left|\xi_{1}\right|^{2}+b-a^{2}} \\
& \geq \frac{C\left(t, x_{2}\right)}{\sqrt{1+\left|\xi_{1}\right|^{2}}} .
\end{aligned}
$$

This completes the proof of (a).

(b) For fixed $t>0$, the left-hand side of (3.13) is a continuous and positive function of $\xi_{1}$, which is therefore bounded below by a positive constant for $\left|\xi_{1}\right|$ bounded. On the other hand, the right-hand side of (3.13) is bounded above. Therefore, it suffices to check (3.13) for $\left|\xi_{1}\right|$ sufficiently large. In this case,

$$
\begin{aligned}
\int_{0}^{t} d s \mathcal{F}_{1} G_{1}\left(s, \xi_{1}, 0\right)^{2} & \geq \frac{e^{-2 a^{+} t}}{4} \int_{0}^{t} d s J_{0}^{2}\left(s \sqrt{\left|\xi_{1}\right|^{2}+b-a^{2}}\right) \\
& =\frac{e^{-2 a^{+} t}}{4 \sqrt{\left|\xi_{1}\right|^{2}+b-a^{2}}} \int_{0}^{t \sqrt{\left|\xi_{1}\right|^{2}+b-a^{2}}} d r J_{0}^{2}(r),
\end{aligned}
$$

by the change of variable $r=s \sqrt{\left|\xi_{1}\right|^{2}+b-a^{2}}$. By Lemma C.2, the integral is bounded below by

$$
c \ln \left(t \sqrt{\left|\xi_{1}\right|^{2}+b-a^{2}}\right),
$$

which yields (3.13).

\section{Solutions to the linear S.P.D.E.}

The most classical notion of solution to (2.12) is the notion of weak solution: a weak solution of (2.12) is an adapted process $(u(t), t \geq 0)$ with values in $\mathcal{S}^{\prime}\left(\mathbb{R}^{d}\right)$ 
such that for all $t>0$ and $\varphi \in C_{0}^{\infty}\left(\mathbb{R}^{d}\right)$,

$$
\left\langle\frac{\partial^{2} u(t)}{\partial t^{2}}(\cdot)+2 a \frac{\partial u(t)}{\partial t}(\cdot)+b u(t)(\cdot)-\Delta u(t)(\cdot), \varphi\right\rangle=\langle\dot{F}(t, \cdot), \varphi(\cdot, 0)\rangle, \quad \mathbb{P}-\text { a.s. }
$$

It is well known (see for instance [7, 30, 32]) that the unique weak solution to (2.12) is given by the formula

$$
\langle u(t), \varphi\rangle=\int_{[0, t] \times \mathbb{R}^{d-1}} M\left(d s, d x_{1}\right)(G(t-s) * \varphi)\left(x_{1}, 0\right), \quad t \in \mathbb{R}_{+}, \varphi \in \mathcal{S}\left(\mathbb{R}^{d}\right),
$$

where $G$ is the Green kernel of equation (3.1), whose properties are listed in Section 3.

Remark 4.1. Note that we could intepret the noise term as a boundary term, and therefore consider that (4.1) is the weak solution of the following equation in the upper half space:

$$
\frac{\partial^{2} u}{\partial t^{2}}(t, x)+2 a \frac{\partial u}{\partial t}(t, x)+b u(t, x)-\Delta u(t, x)=0, \quad(t, x) \in \mathbb{R}_{+} \times \mathbb{R}^{d-1} \times \mathbb{R}_{+},
$$

with the stochastic boundary condition

$$
\frac{\partial u}{\partial x_{2}}\left(t, x_{1}, 0\right)=\dot{F}\left(t, x_{1}\right) .
$$

Indeed, this problem leads to the same weak formulation as (4.1).

In many contexts, stronger notions of solution than that in (4.1) are useful. We shall examine two such notions defined in Sections 4.1 and 4.2 below: functionvalued solutions and random field solutions. We shall see that the conditions for existence of a function-valued solution and of a random field solution are not the same!

4.1. Function-valued solutions. A function-valued solution to (2.12) is an adapted process $(u(t), t \geq 0)$ with values in $L_{l o c}^{2}\left(\mathbb{R}^{d}\right)$ such that

$$
\varphi \mapsto \int_{\mathbb{R}^{d}} d x u(t)(x) \varphi(x), \quad \varphi \in C_{0}^{\infty}\left(\mathbb{R}^{d}\right), t \geq 0,
$$

coincides with the weak solution (4.1) of (2.12). This type of solution is often considered in $[9$. Note that for fixed $t \geq 0, u(t)(x)$ need only be defined for almost all $x \in \mathbb{R}^{d}$. We shall show that a function-valued solution exists if and only if the following condition is satisfied.

\section{Assumption $B_{0}$.}

$$
\int_{\mathbb{R}^{d-1}} \frac{\mu\left(d \xi_{1}\right)}{\sqrt{1+\left|\xi_{1}\right|^{2}}}<\infty
$$

A reformulation of this condition into a condition on the covariance of the noise is given in Appendix A. Note that this condition is stronger than that needed for the equation driven by spatially homogeneous noise on $\mathbb{R}^{d}$ (see [5, 17, or even [11]), for which the square root does not appear. This is to be expected since our noise is concentrated on a hyperplane, which makes it more singular than noise spread out over $\mathbb{R}^{d}$. We point out that Lebesgue measure on $\mathbb{R}^{d-1}$ (which is the spectral 
measure of white noise on $\mathbb{R}^{d-1}$ ) does not satisfy this condition for any dimension $d>1$.

We begin by establishing two lemmas.

Lemma 4.2. Under Assumption $B_{0}$ and for $\left(t, x_{1}, x_{2}\right) \in \mathbb{R}_{+} \times \mathbb{R}^{d-1} \times \mathbb{R}^{*}$, the function $\phi_{t, x_{1}, x_{2}}:[0, t] \rightarrow O_{C}^{\prime}\left(\mathbb{R}^{d-1}\right)$ defined by

$$
\phi_{t, x_{1}, x_{2}}(s)=G_{1}\left(t-s, x_{1}-\cdot, x_{2}\right), \quad s \in[0, t]
$$

belongs to $H_{t}$.

Proof. Notice that

$$
\mathcal{F}_{1} \phi_{t, x_{1}, x_{2}}\left(s, \xi_{1}\right)=\mathcal{F}_{1} G_{1}\left(t-s,-\xi_{1}, x_{2}\right) \chi_{x_{1}}\left(\xi_{1}\right) .
$$

Thus, for all $s \in[0, t], \mathcal{F}_{1} \phi_{t, x_{1}, x_{2}}(s, \cdot) \in O_{M}\left(\mathbb{R}^{d-1}\right)$ (see Section 2.1) and this implies that $\phi_{t, x_{1}, x_{2}}(s, \cdot) \in O_{C}^{\prime}\left(\mathbb{R}^{d-1}\right)$, by (2.3). Moreover, $\mathcal{F}_{1} \phi_{t, x_{1}, x_{2}}$ is a Borel-measurable function and using Lemma 3.4(a) and Assumption $B_{0}$, we obtain

$$
\begin{aligned}
\left\|\phi_{t, x_{1}, x_{2}}\right\|_{t}^{2} & =\int_{\mathbb{R}^{d-1}} \mu\left(d \xi_{1}\right) \int_{0}^{t} d s \mathcal{F}_{1} G_{1}\left(t-s,-\xi_{1}, x_{2}\right)^{2} \\
& \leq C(t) \int_{\mathbb{R}^{d-1}} \frac{\mu\left(d \xi_{1}\right)}{\sqrt{1+\left|\xi_{1}\right|^{2}}} \operatorname{arccosh}\left(\frac{t}{\left|x_{2}\right|}\right) 1_{\left\{\left|x_{2}\right|<t\right\}}<\infty,
\end{aligned}
$$

since $x_{2} \neq 0$. With the change of variables $s \rightarrow t-s$, (2.21) is implied by

$$
\lim _{h \downarrow 0} \int_{0}^{t} d s \int_{\mathbb{R}^{d-1}} \mu\left(d \xi_{1}\right) \sup _{s<r<s+h}\left|\mathcal{F}_{1} G_{1}\left(r,-\xi_{1}, x_{2}\right)-\mathcal{F}_{1} G_{1}\left(s, \xi_{1}, x_{2}\right)\right|^{2}=0 .
$$

Fix $\xi_{1}$ and $s \neq\left|x_{2}\right|$. If $s<\left|x_{2}\right|$, then for $h$ sufficiently small and $\left.r \in\right] s, s+h[$, $r<\left|x_{2}\right|$, so the integrand in (4.3) vanishes. For $s>\left|x_{2}\right|$ and $\left.r \in\right] s, s+h\left[, r>\left|x_{2}\right|\right.$, so the integrand is equal to

$$
\begin{aligned}
\sup _{s<r<s+h} \mid e^{-a s} J\left(\sqrt{\left(\left|\xi_{1}\right|^{2}+b-a^{2}\right)\left(r^{2}-x_{2}^{2}\right)}\right) & \\
& -\left.e^{-a r} J\left(\sqrt{\left(\left|\xi_{1}\right|^{2}+b-a^{2}\right)\left(s^{2}-x_{2}^{2}\right)}\right)\right|^{2},
\end{aligned}
$$

where $J(r)$ is either $J_{0}(r)$ or $I_{0}(i r)$. By uniform continuity of $J$, this expression converges to 0 as $h \downarrow 0$. Therefore, the integrand in (4.3) converges pointwise to 0 , for a.a. $\xi_{1}$ and $s$.

In order to apply the dominated convergence theorem, we note from Lemma 3.3 that

$$
\begin{aligned}
& \sup _{s<r<s+h}\left|\mathcal{F}_{1} G_{1}\left(r,-\xi_{1}, x_{2}\right)-\mathcal{F}_{1} G_{1}\left(s,-\xi_{1}, x_{2}\right)\right|^{2} \\
& \leq \frac{C}{\sqrt{1+\left|\xi_{1}\right|^{2}}} 1_{\left\{\left|x_{2}\right|<s\right\}} \sup _{s<r<s+h}\left(\frac{1}{\sqrt{r^{2}-\left|x_{2}^{2}\right|}}+\frac{1}{\sqrt{s^{2}-\left|x_{2}^{2}\right|}}\right) \\
& \leq \frac{C}{\sqrt{1+\left|\xi_{1}\right|^{2}}} \frac{2}{\sqrt{s^{2}-\left|x_{2}^{2}\right|}} 1_{\left\{\left|x_{2}\right|<s\right\}} .
\end{aligned}
$$

By Assumption $B_{0}$, this last expression is $d s \times \mu\left(d \xi_{1}\right)$-integrable, so the dominated convergence theorem applies and (4.3) is proved. The conclusion now follows from Theorem 2.3. 
Lemma 4.3. Let $M$ be the worthy martingale measure defined in Section 2.5. Under Assumption $B_{0}$, the real-valued process $X=\left\{X\left(t, x_{1}, x_{2}\right),\left(t, x_{1}, x_{2}\right) \in \mathbb{R}_{+} \times\right.$ $\left.\mathbb{R}^{d-1} \times \mathbb{R}^{*}\right\}$ defined by

$$
\begin{aligned}
X\left(t, x_{1}, x_{2}\right)=\int_{[0, t] \times \mathbb{R}^{d-1}} M\left(d s, d y_{1}\right) G_{1}\left(t-s, x_{1}-y_{1}, x_{2}\right), & \\
\left(t, x_{1}, x_{2}\right) & \in \mathbb{R}_{+} \times \mathbb{R}^{d-1} \times \mathbb{R}^{*},
\end{aligned}
$$

is a centered Gaussian process whose covariance is given by

$$
\begin{aligned}
& \mathbb{E}\left(X\left(t, x_{1}, x_{2}\right) X\left(s, y_{1}, y_{2}\right)\right) \\
& \quad=\int_{\mathbb{R}^{d-1}} \mu\left(d \xi_{1}\right) \int_{0}^{t \wedge s} d r \mathcal{F}_{1} G_{1}\left(t-r,-\xi_{1}, x_{2}\right) \mathcal{F}_{1} G_{1}\left(s-r,-\xi_{1}, y_{2}\right) \chi_{x_{1}-y_{1}}\left(\xi_{1}\right),
\end{aligned}
$$

and such that the map $\left(t, x_{1}, x_{2}\right) \mapsto X\left(t, x_{1}, x_{2}\right)$ is continuous from $\mathbb{R}_{+} \times \mathbb{R}^{d-1} \times \mathbb{R}^{*}$ to $L^{2}(\Omega)$.

Remark 4.4. This result and [23, Prop. 3.6 and Cor. 3.8] imply that the process $X$ admits a modification $\tilde{X}$ such that the map $\left(t, x_{1}, x_{2}, \omega\right) \mapsto \tilde{X}\left(t, x_{1}, x_{2}, \omega\right)$ is jointly measurable. We will implicitly consider this modification in the following.

Proof. of Lemma 4.3. By Lemma 4.2, the process $X$ is well defined. The fact that $X$ is a centered Gaussian process with the covariance given in (4.4) follows easily from the isometry (2.20), and since $\mu$ and $\mathcal{F}_{1} G$ are symmetric in $\xi_{1}$, (4.4) is equal to

$$
\int_{\mathbb{R}^{d-1}} \mu\left(d \xi_{1}\right) \int_{0}^{t \wedge s} d r \mathcal{F}_{1} G_{1}\left(t-r,-\xi_{1}, x_{2}\right) \mathcal{F}_{1} G_{1}\left(s-r,-\xi_{1}, y_{2}\right) \cos \left(\xi_{1} \cdot\left(x_{1}-y_{1}\right)\right),
$$

so $X$ is real-valued.

In order to show that the map $\left(t, x_{1}, x_{2}\right) \mapsto X\left(t, x_{1}, x_{2}\right)$ is continuous from $\mathbb{R}_{+} \times \mathbb{R}^{d-1} \times \mathbb{R}^{*}$ to $L^{2}(\Omega)$, we show that for all $T>0$, it is continuous from $[0, T] \times$ $\mathbb{R}^{d-1} \times \mathbb{R}^{*}$ to $L^{2}(\Omega)$, showing first that the map $x_{2} \mapsto X\left(t, x_{1}, x_{2}\right)$ is continuous in $L^{2}(\Omega)$ uniformly in $\left(t, x_{1}\right) \in[0, T] \times \mathbb{R}^{d-1}$, then that for fixed $x_{2} \in \mathbb{R}^{*}$, the map $x_{1} \mapsto X\left(t, x_{1}, x_{2}\right)$ is continuous in $L^{2}(\Omega)$ uniformly in $t \in[0, T]$ and finally that for fixed $\left(x_{1}, x_{2}\right) \in \mathbb{R}^{d-1} \times \mathbb{R}^{*}$, the map $t \mapsto X\left(t, x_{1}, x_{2}\right)$ is continuous in $L^{2}(\Omega)$.

Let $x_{2}, y_{2} \in \mathbb{R}^{*}$. Using the isometry (2.20) (or (4.4)), we obtain

$$
\begin{aligned}
& \sup _{\left(t, x_{1}\right) \in[0, T] \times \mathbb{R}^{d-1}} \mathbb{E}\left(\left(X\left(t, x_{1}, y_{2}\right)-X\left(t, x_{1}, x_{2}\right)\right)^{2}\right) \\
& \leq \int_{\mathbb{R}^{d-1}} \mu\left(d \xi_{1}\right) \int_{0}^{T} d r\left(\mathcal{F}_{1} G_{1}\left(r,-\xi_{1}, y_{2}\right)-\mathcal{F}_{1} G_{1}\left(r,-\xi_{1}, x_{2}\right)\right)^{2} .
\end{aligned}
$$

We will show that this expression converges to 0 as $y_{2} \rightarrow x_{2}$. First note that for each $\xi_{1} \in \mathbb{R}^{d-1}$ and $r \neq\left|x_{2}\right|$,

$$
\left(\mathcal{F}_{1} G_{1}\left(r,-\xi_{1}, y_{2}\right)-\mathcal{F}_{1} G_{1}\left(r,-\xi_{1}, x_{2}\right)\right)^{2} \underset{y_{2} \rightarrow x_{2}}{\rightarrow} 0 .
$$

Moreover, since $\mathcal{F}_{1} G_{1}$ is bounded on $[0, T] \times \mathbb{R}^{d-1} \times \mathbb{R}$, we obtain from the dominated convergence theorem that

$$
\int_{0}^{T} d r\left(\mathcal{F}_{1} G_{1}\left(r,-\xi_{1}, y_{2}\right)-\mathcal{F}_{1} G_{1}\left(r,-\xi_{1}, x_{2}\right)\right)^{2} \underset{y_{2} \rightarrow x_{2}}{\rightarrow} 0 .
$$


But for $\varepsilon \in] 0,\left|x_{2}\right|\left[\right.$ and $\left|y_{2}-x_{2}\right|<\varepsilon$, we obtain by Lemma 3.4(a) that

$$
\begin{array}{rl}
\int_{0}^{T} & d r\left(\mathcal{F}_{1} G_{1}\left(r,-\xi_{1}, y_{2}\right)-\mathcal{F}_{1} G_{1}\left(r,-\xi_{1}, x_{2}\right)\right)^{2} \\
\leq & \frac{2 C(T)}{\sqrt{1+\left|\xi_{1}\right|^{2}}}\left(\operatorname{arccosh}\left(\frac{T}{\left|y_{2}\right|}\right) 1_{\left\{\left|y_{2}\right|<T\right\}}+\operatorname{arccosh}\left(\frac{T}{\left|x_{2}\right|}\right) 1_{\left\{\left|x_{2}\right|<T\right\}}\right) \\
\leq & \frac{2 C(T)}{\sqrt{1+\left|\xi_{1}\right|^{2}}}\left(\operatorname{arccosh}\left(\frac{T}{\left|x_{2}\right|-\varepsilon}\right) 1_{\left\{\left|x_{2}\right|-\varepsilon<T\right\}}\right. \\
\left.+\operatorname{arccosh}\left(\frac{T}{\left|x_{2}\right|}\right) 1_{\left\{\left|x_{2}\right|<T\right\}}\right)
\end{array}
$$

since $\left|y_{2}\right|>\left|x_{2}\right|-\epsilon$. Therefore, by Assumption $B_{0}$ and the dominated convergence theorem, (4.5) converges to 0 as $y_{2} \rightarrow x_{2}$.

Now, let $x_{1}, y_{1} \in \mathbb{R}^{d-1}$ and $x_{2} \in \mathbb{R}^{*}$. By (4.4), we have

$$
\begin{aligned}
& \sup _{t \in[0, T]} \mathbb{E}\left(\left(X\left(t, y_{1}, x_{2}\right)-X\left(t, x_{1}, x_{2}\right)\right)^{2}\right) \\
& \quad \leq \int_{\mathbb{R}^{d-1}} \mu\left(d \xi_{1}\right) \int_{0}^{T} d r \mathcal{F}_{1} G_{1}\left(r,-\xi_{1}, x_{2}\right)^{2} 2\left(1-\cos \left(\xi_{1} \cdot\left(y_{1}-x_{1}\right)\right)\right) .
\end{aligned}
$$

The integrand in (4.7) converges to 0 as $y_{1} \rightarrow x_{1}$, so for fixed $\xi_{1} \in \mathbb{R}^{d-1}$, the inner integral does too since $\mathcal{F}_{1} G_{1}$ is bounded on $[0, T] \times \mathbb{R}^{d-1} \times \mathbb{R}$. By Lemma 3.4(a), which applies since $x_{2} \neq 0$ and Assumption $B_{0}$ holds, we conclude from the dominated convergence theorem that the expresssion in (4.7) converges to 0 as $y_{1} \rightarrow x_{1}$.

Finally, let $t, h \in \mathbb{R}_{+}, x_{1} \in \mathbb{R}^{d-1}$ and $x_{2} \in \mathbb{R}^{*}$. From (4.4), we obtain

$$
\begin{aligned}
& \mathbb{E}\left(\left(X\left(t+h, x_{1}, x_{2}\right)-X\left(t, x_{1}, x_{2}\right)\right)^{2}\right) \\
& =\int_{\mathbb{R}^{d-1}} \mu\left(d \xi_{1}\right) \int_{0}^{t} d r\left(\mathcal{F}_{1} G_{1}\left(r+h,-\xi_{1}, x_{2}\right)-\mathcal{F}_{1} G_{1}\left(r,-\xi_{1}, x_{2}\right)\right)^{2} \\
& \quad+\int_{\mathbb{R}^{d-1}} \mu\left(d \xi_{1}\right) \int_{0}^{h} d q \mathcal{F}_{1} G_{1}\left(q,-\xi_{1}, x_{2}\right)^{2} .
\end{aligned}
$$

By (4.3), the expression in (4.8) converges to 0 as $h \rightarrow 0$. On the other hand, from Assumption $B_{0}$ and Lemma 3.4(a), which applies since $x_{2} \neq 0$, the integral in (4.9) converges also to 0 as $h \rightarrow 0$, and this shows the right-continuity in $t$ of the process $X$ (in $L^{2}(\Omega)$ ). The left-continuity follows in the same way, and this completes the proof.

With these two lemmas in hand, we can now prove the following theorem.

Theorem 4.5. There exists a function-valued solution $(u(t), t \geq 0)$ to equation (2.12) if and only if Assumption $B_{0}$ is satisfied. In this case, $u(t)\left(x_{1}, x_{2}\right)=$ $X\left(t, x_{1}, x_{2}\right), x_{2} \neq 0, x_{1} \in \mathbb{R}^{d-1}$, is the function-valued solution, where $\left(X\left(t, x_{1}, x_{2}\right)\right)$ is defined in Lemma 4.3 .

Proof. Let us first suppose that Assumption $B_{0}$ is satisfied. Let $X$ be the process defined in Lemma4.3. it is continuous in $L^{2}(\Omega)$ on $\mathbb{R}_{+} \times \mathbb{R}^{d-1} \times \mathbb{R}^{*}$ and is a centered 
Gaussian process whose covariance is given by (4.4). Set $u(t)\left(x_{1}, x_{2}\right)=X\left(t, x_{1}, x_{2}\right)$, $x_{2} \neq 0, x_{1} \in \mathbb{R}^{d}$. Observe that for fixed $t \in \mathbb{R}_{+}$, we have by (4.4),

$$
\mathbb{E}\left(X\left(t, x_{1}, x_{2}\right)^{2}\right)=\int_{\mathbb{R}^{d-1}} \mu\left(d \xi_{1}\right) \int_{0}^{t} d s \mathcal{F}_{1} G_{1}\left(t-s,-\xi_{1}, x_{2}\right)^{2} .
$$

By Lemma 3.4(a) and Assumption $B_{0}$, this is bounded above by

$$
C(t) \operatorname{arccosh}\left(\frac{t}{\left|x_{2}\right|}\right) 1_{\left\{\left|x_{2}\right|<t\right\}} \underset{x_{2} \rightarrow 0}{\sim} \ln \left(\frac{1}{\left|x_{2}\right|}\right) \text {. }
$$

This is integrable in the neighborhood of $x_{2}=0$, so a.s., $u(t) \in L_{l o c}^{2}\left(\mathbb{R}^{d}\right)$.

We now prove that for $\varphi \in \mathcal{S}\left(\mathbb{R}^{d}\right)$,

$$
\langle u(t), \varphi\rangle=\int_{\mathbb{R}^{d}} d x u(t)(x) \varphi(x), \quad \mathbb{P}-\text { a.s. }
$$

where $\langle u(t), \varphi\rangle$ denotes the right-hand side of (4.1). By Lemma 4.3 and Remark 4.4. the integral on the right-hand side of (4.11) is well defined. We show that both sides of (4.11) are equal $\mathbb{P}$-a.s., by showing that both their variances are equal to their covariance. By (4.1) and (2.20),

$$
\begin{aligned}
\mathbb{E}\left(|\langle u(t), \varphi\rangle|^{2}\right) & =\mathbb{E}\left(\left|\int_{[0, t] \times \mathbb{R}^{d-1}} M\left(d s, d x_{1}\right)(G(t-s) * \varphi)\left(x_{1}, 0\right)\right|^{2}\right) \\
& =\int_{\mathbb{R}^{d-1}} \mu\left(d \xi_{1}\right) \int_{0}^{t} d s\left|\mathcal{F}_{1}(G(t-s) * \varphi)\left(\xi_{1}, 0\right)\right|^{2} .
\end{aligned}
$$

Since $\mathcal{F}_{1}=\mathcal{F}_{2}^{-1} \mathcal{F}$ and $\mathcal{F}(G * H)=\mathcal{F} G \cdot \mathcal{F} H$

$$
\begin{aligned}
\mathcal{F}_{1}(G(t-s) * \varphi)\left(\xi_{1}, 0\right) & =\mathcal{F}_{2}^{-1}(\mathcal{F} G(t-s) \cdot \mathcal{F} \varphi)\left(\xi_{1}, 0\right) \\
& =\frac{1}{2 \pi} \int_{\mathbb{R}} d \xi_{2} \mathcal{F} G\left(t-s, \xi_{1}, \xi_{2}\right) \mathcal{F} \varphi\left(\xi_{1}, \xi_{2}\right),
\end{aligned}
$$

So

$$
\mathbb{E}\left(|\langle u(t), \varphi\rangle|^{2}\right)=\int_{\mathbb{R}^{d-1}} \mu\left(d \xi_{1}\right) \int_{0}^{t} d s\left|\frac{1}{2 \pi} \int_{\mathbb{R}} d \xi_{2} \mathcal{F} G\left(t-s, \xi_{1}, \xi_{2}\right) \mathcal{F} \varphi\left(\xi_{1}, \xi_{2}\right)\right|^{2} .
$$

On the other hand, by Fubini's theorem and (4.4),

$$
\begin{aligned}
\mathbb{E}\left(\left|\int_{\mathbb{R}^{d}} d x X(t, x) \varphi(x)\right|^{2}\right) \\
=\int_{\mathbb{R}^{d}} d x \int_{\mathbb{R}^{d}} d y \mathbb{E}(X(t, x) X(t, y)) \varphi(x) \overline{\varphi(y)} \\
=\int_{\mathbb{R}^{d-1}} \mu\left(d \xi_{1}\right) \int_{0}^{t} d s \\
\quad \cdot\left|\int_{\mathbb{R}^{d-1}} d x_{1} \int_{\mathbb{R}} d x_{2} \mathcal{F}_{1} G_{1}\left(t-s,-\xi_{1}, x_{2}\right) \chi_{\xi_{1}}\left(x_{1}\right) \varphi\left(x_{1}, x_{2}\right)\right|^{2} .
\end{aligned}
$$

By definition of $G_{1}$, the double integral inside the modulus is equal to (4.15)

$\int_{\mathbb{R}} d x_{2} \mathcal{F}_{1} G_{1}\left(t-s,-\xi_{1}, x_{2}\right) \mathcal{F}_{1} \varphi\left(\xi_{1}, x_{2}\right)=\frac{1}{2 \pi} \int_{\mathbb{R}} d \xi_{2} \mathcal{F} G\left(t-s,-\xi_{1},-\xi_{2}\right) \mathcal{F} \varphi\left(\xi_{1}, \xi_{2}\right)$, 
which is equal to (4.12), so (4.13) and (4.14) are equal. It remains to compute, using Fubini's theorem, (4.1) and (2.20), the covariance

$$
\begin{aligned}
& \mathbb{E}\left(\langle u(t), \varphi\rangle \cdot \overline{\int_{\mathbb{R}^{d}} d x X(t, x) \varphi(x)}\right) \\
&\left.=\int_{\mathbb{R}^{d-1}} \mu\left(d \xi_{1}\right) \int_{0}^{t} \frac{d s\left(\mathcal{F}_{1}(G(t-s) * \varphi)\left(\xi_{1}, 0\right)\right.}{\cdot \int_{\mathbb{R}^{d}} d x \mathcal{F}_{1} G_{1}\left(t-s,-\xi_{1}, x_{2}\right) \chi_{\xi_{1}}\left(x_{1}\right) \varphi(x)}\right)
\end{aligned}
$$

Using calculations (4.12) and (4.15), we obtain that the two factors in the last integrand are equal, so (4.16) is also equal to (4.13) and (4.14). This proves (4.11) and therefore the sufficiency of Assumption $B_{0}$.

Let us now prove the necessity of Assumption $B_{0}$. We assume that there is a process $(u(t), t \geq 0)$ with values in $L_{l o c}^{2}\left(\mathbb{R}^{d}\right)$ that satisfies (4.11). For $\left(t, x_{1}, x_{2}\right) \in$ $\mathbb{R}_{+} \times \mathbb{R}^{d-1} \times \mathbb{R}$ such that $\left|x_{2}\right|<t$, let $\varphi_{x_{1}, x_{2}}^{(n)}=\delta_{\left(x_{1}, x_{2}\right)} * \psi_{n} \in \mathcal{S}\left(\mathbb{R}^{d}\right)$, where $\left(\psi_{n}\right)$ is a sequence of non-negative and compactly supported approximations of $\delta_{0}$ in $\mathbb{R}^{d}$. The assumptions made on $u$ and Fubini's theorem imply that

$$
\begin{gathered}
\mathbb{E}\left(\left|\left\langle u(t), \varphi_{x_{1}, x_{2}}^{(n)}\right\rangle\right|^{2}\right)=\mathbb{E}\left(\left|\int_{\mathbb{R}^{d}} d y_{1} d y_{2} u(t)\left(y_{1}, y_{2}\right) \varphi_{x_{1}, x_{2}}^{(n)}\left(y_{1}, y_{2}\right)\right|^{2}\right) \\
=\int_{\mathbb{R}^{d}} d y_{1} d y_{2} \int_{\mathbb{R}^{d}} d z_{1} d z_{2} \mathbb{E}\left(u(t)\left(y_{1}, y_{2}\right) u(t)\left(z_{1}, z_{2}\right)\right) \\
\cdot \varphi_{x_{1}, x_{2}}^{(n)}\left(y_{1}, y_{2}\right) \overline{\varphi_{x_{1}, x_{2}}^{(n)}\left(z_{1}, z_{2}\right)}
\end{gathered}
$$

By the Lebesgue Differentiation Theorem [31, Chap. 7, Exercise 2], this converges as $n \rightarrow \infty$ for a.a. $x$ to $\mathbb{E}\left(u(t)(x)^{2}\right)<\infty$.

On the other hand, replacing $\varphi$ by $\varphi_{x_{1}, x_{2}}^{(n)}$ in (4.13) gives

$$
\begin{aligned}
& \mathbb{E}\left(\left|\left\langle u(t), \varphi_{x_{1}, x_{2}}^{(n)}\right\rangle\right|^{2}\right) \\
& \quad=\int_{\mathbb{R}^{d-1}} \mu\left(d \xi_{1}\right) \int_{0}^{t} d s\left|\frac{1}{2 \pi} \int_{\mathbb{R}} d \xi_{2} \mathcal{F} G\left(t-s, \xi_{1}, \xi_{2}\right) \mathcal{F} \varphi_{x_{1}, x_{2}}^{(n)}\left(\xi_{1}, \xi_{2}\right)\right|^{2}
\end{aligned}
$$

Let us then compute

$$
\begin{aligned}
\frac{1}{2 \pi} & \int_{\mathbb{R}} d \xi_{2} \mathcal{F} G\left(t-s, \xi_{1}, \xi_{2}\right) \mathcal{F} \varphi_{x_{1}, x_{2}}^{(n)}\left(\xi_{1}, \xi_{2}\right) \\
& =\int_{\mathbb{R}} d y_{2} \mathcal{F}_{1} G_{1}\left(t-s, \xi_{1},-y_{2}\right) \mathcal{F}_{1} \varphi_{x_{1}, x_{2}}^{(n)}\left(\xi_{1}, y_{2}\right) \\
& =\int_{\mathbb{R}^{d}} d y_{1} \int_{\mathbb{R}} d y_{2} \mathcal{F}_{1} G_{1}\left(t-s, \xi_{1},-y_{2}\right) \chi_{\xi_{1}}\left(y_{1}\right) \varphi_{x_{1}, x_{2}}^{(n)}\left(y_{1}, y_{2}\right) \\
& \rightarrow \underset{n \rightarrow \infty}{\rightarrow} \mathcal{F}_{1} G_{1}\left(t-s, \xi_{1},-x_{2}\right) \chi_{x_{1}}\left(\xi_{1}\right),
\end{aligned}
$$


for all $\left(s, \xi_{1}\right) \in[0, t] \times \mathbb{R}^{d-1}$ such that $s \neq t-\left|x_{2}\right|$. Fatou's lemma and Lemma 3.5(a) then imply that

$$
\begin{aligned}
\liminf _{n \rightarrow \infty} \mathbb{E}\left(\left|\left\langle u(t), \varphi_{x_{1}, x_{2}}^{(n)}\right\rangle\right|^{2}\right) & \geq \int_{\mathbb{R}^{d-1}} \mu\left(d \xi_{1}\right) \int_{0}^{t} d s \mathcal{F}_{1} G_{1}\left(t-s, \xi_{1},-x_{2}\right)^{2} \\
& \geq C_{6}\left(t, x_{2}\right) \int_{\mathbb{R}^{d-1}} \frac{\mu\left(d \xi_{1}\right)}{\sqrt{1+\left|\xi_{1}\right|^{2}}} .
\end{aligned}
$$

Since the above liminf is a finite limit for a.a. $\left(x_{1}, x_{2}\right)$ by (4.17), Assumption $B_{0}$ is satisfied, and this completes the proof.

Remark 4.6. If $\mathbb{E}\left(X\left(t, x_{1}, x_{2}\right)^{2}\right)$ behaves as $\ln \left(\frac{1}{\left|x_{2}\right|}\right)$ for $x_{2} \rightarrow 0$, as (4.10) suggests, then the process $X$ cannot be continuously extended to the hyperplane $x_{2}=0$. In the next section, we shall see that a stronger assumption on the spectral measure $\mu$ is needed to ensure that a continuous extension to the hyperplane $x_{2}=0$ is possible.

4.2. Random field solutions. In this section, we consider a third notion of solution: a random field solution to (2.12) is an adapted process $(u(t, x),(t, x) \in$ $\left.\mathbb{R}_{+} \times \mathbb{R}^{d}\right)$ such that $(t, x) \mapsto u(t, x)$ is continuous from $\mathbb{R}_{+} \times \mathbb{R}^{d}$ into $L^{2}(\Omega)$, and

$$
\varphi \mapsto \int_{\mathbb{R}^{d}} d x u(t, x) \varphi(x), \quad \varphi \in C_{0}^{\infty}\left(\mathbb{R}^{d}\right), t \geq 0,
$$

coincides with the weak solution of (2.12).

Note that if $(u(t), t \geq 0)$ is a function-valued solution, then $u(t)(x)$ is only defined for a.a. $x$, and there is no continuity requirement on the map $x \mapsto u(t)(x)$, whereas such a continuity requirement is specified in the notion of random field solution. We shall show that the existence of a random field solution is equivalent to the following condition on the spectral measure $\mu$, which is stronger than Assumption $B_{0}$.

\section{Assumption $B_{0}^{\prime}$.}

$$
\int_{\mathbb{R}^{d-1}} \mu\left(d \xi_{1}\right) \frac{\ln \left(1+\left|\xi_{1}\right|^{2}\right)}{\sqrt{1+\left|\xi_{1}\right|^{2}}}<\infty
$$

Note the extra logarithmic factor in this assumption compared to Assumption $B_{0}$. In Example 4.9 below, we give an example of a spectral measure $\mu$ which does not satisfy Assumption $B_{0}^{\prime}$ but does satisfy Assumption $B_{0}$.

We shall need the following lemma, which is analogous to Lemma 4.2. The crucial distinctions are that Assumption $B_{0}$ is replaced by Assumption $B_{0}^{\prime}$ and the conclusion is now valid even for $x_{2}=0$.

Lemma 4.7. Under Assumption $B_{0}^{\prime}$ and for $\left(t, x_{1}, x_{2}\right) \in \mathbb{R}_{+} \times \mathbb{R}^{d-1} \times \mathbb{R}$, the function $\phi_{t, x_{1}, x_{2}}:[0, t] \rightarrow O_{C}^{\prime}\left(\mathbb{R}^{d-1}\right)$ defined by

$$
\phi_{t, x_{1}, x_{2}}(s)=G_{1}\left(t-s, x_{1}-\cdot, x_{2}\right), \quad s \in[0, t],
$$

belongs to $H_{t}$.

Proof. We shall show that $\phi_{t, x_{1}, x_{2}} \in H_{t}$ by using the definition of this space (see the end of Section 2.66). Therefore fix $\left(t, x_{1}, x_{2}\right) \in \mathbb{R}_{+} \times \mathbb{R}^{d-1} \times \mathbb{R}$. As in the proof of Lemma 4.2. note that for all $s \in[0, t]$ and $\xi_{1} \in \mathbb{R}^{d-1}$,

$$
\mathcal{F}_{1} \phi_{t, x_{1}, x_{2}}\left(s, \xi_{1}\right)=\mathcal{F}_{1} G_{1}\left(t-s,-\xi_{1}, x_{2}\right) \chi_{x_{1}}\left(\xi_{1}\right),
$$


and that $\phi_{t, x_{1}, x_{2}}(s, \cdot) \in O_{C}^{\prime}\left(\mathbb{R}^{d-1}\right)$. Moreover, $\mathcal{F}_{1} \phi_{t, x_{1}, x_{2}}$ is a Borel-measurable function and using Lemma 3.4(b) and Assumption $B_{0}^{\prime}$, we obtain

$$
\begin{aligned}
\left\|\phi_{t, x_{1}, x_{2}}\right\|_{t}^{2} & =\int_{\mathbb{R}^{d-1}} \mu\left(d \xi_{1}\right) \int_{0}^{t} d s \mathcal{F}_{1} G_{1}\left(t-s,-\xi_{1}, x_{2}\right)^{2} \\
& \leq C(t) \int_{\mathbb{R}^{d-1}} \mu\left(d \xi_{1}\right) \frac{1+\ln \left(1+\left|\xi_{1}\right|^{2}\right)}{\sqrt{1+\left|\xi_{1}\right|^{2}}}<\infty .
\end{aligned}
$$

Let us now define

$$
\phi_{t, x_{1}, x_{2}}^{(n)}\left(s, y_{1}\right)=\left(\phi_{t, x_{1}, x_{2}}(s) *_{1} \psi_{n}\right)\left(y_{1}\right), \quad s \in[0, t], y_{1} \in \mathbb{R}^{d-1},
$$

where $\left(\psi_{n}\right)$ is a sequence of non-negative approximations of $\delta_{0}$ in $\mathbb{R}^{d-1}$, compactly supported in $B_{d-1}(0,1)$ and which satisfy $\int_{\mathbb{R}^{d-1}} d x_{1} \psi_{n}\left(x_{1}\right)=1$. In particular, $\lim _{n \rightarrow \infty} \mathcal{F}_{1} \psi_{n}\left(\xi_{1}\right)=1$ and $\left|\mathcal{F}_{1} \psi_{n}\left(\xi_{1}\right)\right| \leq 1$, for all $\xi_{1} \in \mathbb{R}^{d-1}$.

For each $n$,

$$
\mathcal{F}_{1} \phi_{t, x_{1}, x_{2}}^{(n)}\left(s, \xi_{1}\right)=\mathcal{F}_{1} \phi_{t, x_{1}, x_{2}}\left(s, \xi_{1}\right) \mathcal{F}_{1} \psi_{n}\left(\xi_{1}\right),
$$

which implies that

$$
\left\|\phi_{t, x_{1}, x_{2}}-\phi_{t, x_{1}, x_{2}}^{(n)}\right\|_{t}^{2}=\int_{0}^{t} d s \int_{\mathbb{R}^{d-1}} \mu\left(d \xi_{1}\right)\left|\mathcal{F}_{1} \phi_{t, x_{1}, x_{2}}\left(s, \xi_{1}\right)\right|^{2}\left|1-\mathcal{F}_{1} \psi_{n}\left(\xi_{1}\right)\right|^{2} .
$$

Using the dominated convergence theorem, which applies since the integrand converges to $0,\left|1-\mathcal{F}_{1} \psi_{n}\left(\xi_{1}\right)\right| \leq 2$ and $\left\|\phi_{t, x_{1}, x_{2}}\right\|_{t}<\infty$, we conclude that

$$
\lim _{n \rightarrow \infty}\left\|\phi_{t, x_{1}, x_{2}}-\phi_{t, x_{1}, x_{2}}^{(n)}\right\|_{t}=0 .
$$

It remains to check that $\phi_{t, x_{1}, x_{2}}^{(n)} \in H_{t, 0}$ for each $n$. By (4.21) and definition of $\phi_{t, x_{1}, x_{2}}^{(n)}, \mathcal{F}_{1} \phi_{t, x_{1}, x_{2}}^{(n)}$ is a Borel-measurable function and for all $s \in[0, t], \phi_{t, x_{1}, x_{2}}^{(n)}(s, \cdot) \in$ $\mathcal{S}\left(\mathbb{R}^{d-1}\right)$, since $\phi_{t, x_{1}, x_{2}}(s, \cdot) \in O_{C}^{\prime}\left(\mathbb{R}^{d-1}\right)$. The last condition to be verified is that $\left\|\phi_{t, x_{1}, x_{2}}^{(n)}\right\|_{t,+}<\infty$.

The definition of $\phi_{t, x_{1}, x_{2}}^{(n)}$ implies that for all $s \in[0, t], \phi_{t, x_{1}, x_{2}}^{(n)}(s, \cdot)$ is compactly supported, and therefore belongs to $C_{0}^{\infty}\left(\mathbb{R}^{d-1}\right)$ and so does $\phi_{t, x_{1}, x_{2}}^{(n)}(s, \cdot) *_{1}$ $\phi_{t, x_{1}, x_{2}}^{(n)}(s, \cdot)$. Moreover, since $\operatorname{supp} G\left(s, x_{1}-\cdot, x_{2}\right) \subset B_{d-1}\left(0,\left|x_{1}\right|+t\right)$ for all $s \in[0, t]$ and $x_{2} \in \mathbb{R}$, we obtain that for $R=2\left(\left|x_{1}\right|+t+1\right)$,

$$
\sup _{s \in[0, t]}\left(\left|\phi_{t, x_{1}, x_{2}}^{(n)}(s, \cdot)\right| *_{1}\left|\phi_{t, x_{1}, x_{2}}^{(n)}(s, \cdot)\right|\right)\left(z_{1}\right)=0, \quad \text { for all } z_{1} \in \mathbb{R}^{d-1} \text { with }\left|z_{1}\right|>R \text {. }
$$

This implies that

$$
\left\|\phi_{t, x_{1}, x_{2}}^{(n)}\right\|_{t,+}^{2}=\int_{0}^{t} d s \int_{\mathbb{R}^{d-1}} \nu\left(d z_{1}\right)\left(\left|\phi_{t, x_{1}, x_{2}}^{(n)}(s, \cdot)\right| *_{1} \mid \phi_{t, x_{1}, x_{2}}^{(n)}(s, \cdot)\right) \mid\left(z_{1}\right)<\infty,
$$

which completes the proof.

Theorem 4.8. There exists a random field solution $\left(u(t, x),(t, x) \in \mathbb{R}_{+} \times \mathbb{R}^{d}\right)$ to (2.12) if and only if Assumption $B_{0}^{\prime}$ is satisfied. In this case, $u\left(t, x_{1}, x_{2}\right)=$ $X\left(t, x_{1}, x_{2}\right)$ is the random field solution to (2.12), where $X$ is defined as in Lemma 4.3, but for all $(t, x) \in \mathbb{R}_{+} \times \mathbb{R}^{d}$. 
Proof. Suppose that Assumption $B_{0}^{\prime}$ in satisfied. Then the statement of Lemma 4.3 remains valid for all $x_{2}$, though the proof must be modified as follows. First, by Lemma 4.7. the process $X$ is well defined for all $x_{2} \in \mathbb{R}$ and $x_{1} \in \mathbb{R}^{d-1}$, and (4.4) holds.

The $L^{2}$-increments of $X$ can be estimated as follows. Let us first consider $x_{2}$, $y_{2} \in \mathbb{R}$. We express

$$
\sup _{\left(t, x_{1}\right) \in[0, T] \times \mathbb{R}^{d-1}} \mathbb{E}\left(\left(X\left(t, x_{1}, y_{2}\right)-X\left(t, x_{1}, x_{2}\right)\right)^{2}\right)
$$

as in (4.5). Using the dominated convergence theorem twice as in the proof of Lemma 4.3, but applying this time Lemma 3.4(b) and Assumption $B_{0}^{\prime}$ for (4.6), we obtain that the above expression converges to 0 as $y_{2} \rightarrow x_{2}$.

Now, let $x_{1}, y_{1} \in \mathbb{R}^{d-1}$ and $x_{2} \in \mathbb{R}$. We express

$$
\sup _{t \in[0, T]} \mathbb{E}\left(\left(X\left(t, y_{1}, x_{2}\right)-X\left(t, x_{1}, x_{2}\right)\right)^{2}\right)
$$

as in (4.7). Once again, using the dominated convergence theorem twice jointly with Lemma 3.4 (b) and Assumption $B_{0}^{\prime}$, we obtain that this expression converges to 0 as $y_{1} \rightarrow x_{1}$.

Finally, let $t, h \in \mathbb{R}_{+}, x_{1} \in \mathbb{R}^{d-1}$ and $x_{2} \in \mathbb{R}$. We write

$$
\mathbb{E}\left(\left(X\left(t+h, x_{1}, x_{2}\right)-X\left(t, x_{1}, x_{2}\right)\right)^{2}\right)
$$

as in (4.8) and (4.9). Since $\mathcal{F}_{1} G_{1}$ is bounded on $[0, T] \times \mathbb{R}^{d-1} \times \mathbb{R}$, the inner integral in these equations converges to 0 as $h \rightarrow 0$. We then use Lemma 3.4(b) and Assumption $B_{0}^{\prime}$ to conclude via the dominated convergence theorem that these expressions converge to 0 as $h \rightarrow 0$.

A similar argument allows us to prove the left-continuity. Summing up these results gives us the $L^{2}$-continuity of the process $X$ on $\mathbb{R}_{+} \times \mathbb{R}^{d}$, then the existence of a jointly measurable modification.

One now verifies that (4.19) coincides with the weak solution of (2.12): this is identical to the proof of (4.11). Therefore, Assumption $B_{0}^{\prime}$ implies the existence of a random field solution to (2.12).

In order to prove that Assumption $B_{0}^{\prime}$ is necessary, we also follow the proof of Theorem 4.5. Assuming that there is a random field solution $(u(t, x))$ to (2.12), we have

$$
\infty>\mathbb{E}\left(u\left(t, x_{1}, 0\right)^{2}\right)=\lim _{n \rightarrow \infty} \mathbb{E}\left(\left|\left\langle u(t), \varphi_{x_{1}, 0}^{(n)}\right\rangle\right|^{2}\right),
$$

where $\varphi_{x_{1}, 0}^{(n)} \underset{n \rightarrow \infty}{\rightarrow} \delta_{x_{1}, 0}$ in $\mathcal{S}^{\prime}\left(\mathbb{R}^{d}\right)$. Using (4.13), we obtain that

$$
\begin{aligned}
& \mathbb{E}\left(\left|\left\langle u(t), \varphi_{x_{1}, 0}^{(n)}\right\rangle\right|^{2}\right) \\
& \quad=\int_{\mathbb{R}^{d-1}} \mu\left(d \xi_{1}\right) \int_{0}^{t} d s\left|\frac{1}{2 \pi} \int_{\mathbb{R}} d \xi_{2} \mathcal{F} G\left(t-s, \xi_{1}, \xi_{2}\right) \mathcal{F} \varphi_{x_{1}, 0}^{(n)}\left(\xi_{1}, \xi_{2}\right)\right|^{2} .
\end{aligned}
$$

So by the same calculations as in (4.18) and using Fatou's lemma, we obtain

$$
\lim _{n \rightarrow \infty} \mathbb{E}\left(\left|\left\langle u(t), \varphi_{x_{1}, 0}^{(n)}\right\rangle\right|^{2}\right) \geq \int_{\mathbb{R}^{d-1}} \mu\left(d \xi_{1}\right) \int_{0}^{t} d s \mathcal{F}_{1} G_{1}\left(t-s, \xi_{1}, 0\right)^{2} .
$$

By Lemma 3.5(b),

$$
\int_{\mathbb{R}^{d-1}} \mu\left(d \xi_{1}\right) \int_{0}^{t} d s \mathcal{F}_{1} G_{1}\left(t-s, \xi_{1}, 0\right)^{2} \geq \int_{\mathbb{R}^{d-1}} \mu\left(d \xi_{1}\right) C(t) \frac{\ln \left(1+\left|\xi_{1}\right|^{2}\right)}{\sqrt{1+\left|\xi_{1}\right|^{2}}}
$$


Using (4.22), we conclude that Assumption $B_{0}^{\prime}$ is satisfied, and this completes the proof.

Example 4.9. We give here an example of a spectral measure that satisfies Assumption $B_{0}$ but not Assumption $B_{0}^{\prime}$, for which the function-valued solution to (2.12) cannot therefore be extended continuously to $x_{2}=0$.

Let $d=2$ (so that $\mu$ is a measure on $\mathbb{R}$ ) and describe $\mu$ by its density $\phi$ given by

$$
\phi(r)=3-\frac{2}{e} r \quad \text { if } r \in\left[0, e\left[, \quad \phi(r)=\ln ^{-2}(r) \quad \text { if } r \in[e, \infty[,\right.\right.
$$

and $\phi(r)=\phi(-r)$ for $r<0$. One can easily check that $\mu$ satisfies Assumption $B_{0}$, but not Assumption $B_{0}^{\prime}$.

We now check that the corresponding covariance $\Gamma=\mathcal{F}_{1} \mu$ is a non-negative and non-negative definite measure. Clearly, $\mu$ is a non-negative tempered Borel measure on $\mathbb{R}$, so $\Gamma$ is a tempered non-negative definite distribution by the Bochner-Schwartz theorem [28, Chap. VII, Thm. XVIII]. In order to show that $\Gamma$ is a non-negative measure on $\mathbb{R}$, observe that $\phi$ is continuous, decreasing and convex on $[0, \infty[$, so by Polya's criterion (see for example [13, §2.3.d]), $\phi$ is a (symmetric) non-negative definite function on $\mathbb{R}$. By the classical Bochner theorem, this implies that $\Gamma$ is a non-negative finite measure on $\mathbb{R}$. Therefore, the spectral measure $\mu$ defined above is indeed a relevant example.

Remark 4.10. One can ask about existence of function-valued solutions or random field solutions to the stochastic heat equation driven by noise on a hyperplane, that is,

(4.24)

$$
\left\{\begin{array}{l}
\frac{\partial u}{\partial t}(t, x)-\Delta u(t, x)=\dot{F}\left(t, x_{1}\right) \delta_{0}\left(x_{2}\right), \quad t \in \mathbb{R}_{+}, x=\left(x_{1}, x_{2}\right) \in \mathbb{R}^{d-1} \times \mathbb{R}, \\
u(0, x)=0, \quad x \in \mathbb{R}^{d} .
\end{array}\right.
$$

For this parabolic s.p.d.e., the conclusions are completely different from those of Theorems 4.5] and 4.8 (this contrasts with the spatially homogeneous case [5, 26]). Indeed, in this case,

$$
G(t, x)=\frac{1}{(2 \pi t)^{d / 2}} e^{-|x|^{2} /(2 t)}, \quad G_{1}\left(t, x_{1}, x_{2}\right)=G(t, x),
$$

and

$$
\mathcal{F} G(t, \xi)=e^{-t|\xi|^{2} / 2}, \quad \mathcal{F}_{1} G_{1}\left(t, \xi_{1}, x_{2}\right)=e^{-t\left|\xi_{1}\right|^{2} / 2} \frac{1}{\sqrt{2 \pi t}} e^{-x_{2}^{2} /(2 t)} .
$$

Defining $\phi_{t, x_{1}, x_{2}}(s)$ as in Lemma 4.2, one sees that the integral arising in (4.2) is equal to

$$
\int_{\mathbb{R}^{d-1}} \mu\left(d \xi_{1}\right) \int_{0}^{t} d s e^{-s\left|\xi_{1}\right|^{2}} \frac{1}{2 \pi s} e^{-x_{2}^{2} / s}
$$

When $\left|\xi_{1}\right| \geq 1$, we have

$$
\int_{0}^{t} d s e^{-s\left|\xi_{1}\right|^{2}} \frac{1}{2 \pi s} e^{-x_{2}^{2} / s} \leq \int_{0}^{\infty} d s e^{-s\left|\xi_{1}\right|^{2}} \frac{1}{2 \pi s} e^{-x_{2}^{2} / s}=\frac{1}{\pi} K_{0}\left(2\left|x_{2}\right|\left|\xi_{1}\right|\right),
$$


by [3, formula I.5.34] (where $K_{0}$ is the zero order modified Bessel function of the second kind defined in Appendix (C). When $\left|\xi_{1}\right| \leq 1$ and $x_{2} \neq 0$, we have

$$
\int_{0}^{t} d s e^{-s\left|\xi_{1}\right|^{2}} \frac{1}{2 \pi s} e^{-x_{2}^{2} / s} \leq \int_{0}^{t} d s \frac{1}{2 \pi s} e^{-x_{2}^{2} / s}<\infty .
$$

Using these two estimates, (C.6) and the fact that $\mu$ is a tempered measure, it is not difficult to see that (4.25) is finite for $x_{2} \neq 0$ and therefore that a function-valued solution to (4.24) always exists. This is compatible with the result of Sowers 29].

On the other hand, carrying out the computation in (4.23), one finds that the left-hand side of (4.23) is infinite, no matter the measure $\mu$, because the $d s$-integral is already infinite. It is not difficult to conclude from this that a random field solution to (4.24) never exists.

\section{NON-LINEAR EQUATION DRIVEN BY NOISE ON A HYPERPLANE}

In this section, we shall extend the result of Theorem 4.8 to establish the existence of a random field solution to a non-linear form of equation (2.12). We shall need the following assumption.

\section{Assumption $C_{0}$.}

(i) The covariance $\Gamma$ of the noise is a non-negative measure on $\mathbb{R}^{d-1}$.

(ii) $d \in\{2,3\}$ and $a^{2} \geq b$.

Part (ii) of this assumption implies that the "hyperplane" $x_{2}=0$ is either a straight line or a plane, and from the expressions of $G_{1}$ listed in Appendix B, we see that for all $t \in \mathbb{R}_{+}$and $x_{2} \in \mathbb{R}, G_{1}\left(t, \cdot, x_{2}\right)$ (defined in Proposition 3.1) is a non-negative measure on $\mathbb{R}^{d-1}$. These non-negativity assumptions are needed to use Theorem 2.2, which we shall do repeatedly in the following.

Consider the following formal non-linear equation:

$$
\left\{\begin{array}{l}
\frac{\partial^{2} u}{\partial t^{2}}(t, x)+2 a \frac{\partial u}{\partial t}(t, x)+b u(t, x)-\Delta u(t, x) \\
\quad=g\left(u\left(t, x_{1}, 0\right)\right) \delta_{0}\left(x_{2}\right)+h\left(u\left(t, x_{1}, 0\right)\right) \dot{F}\left(t, x_{1}\right) \delta_{0}\left(x_{2}\right), \quad t \in \mathbb{R}_{+}, x \in \mathbb{R}^{d}, \\
u(0, x)=\frac{\partial u}{\partial t}(0, x)=0, \quad x \in \mathbb{R}^{d},
\end{array}\right.
$$

where $g$ and $h$ are real-valued functions and $\dot{F}$ is the noise concentrated on the hyperplane $x_{2}=0$ considered in Section 2.4. Note that we consider vanishing initial conditions, but this can be improved; see Remark 5.3 .

The integral formulation of (5.1) is

$$
\begin{aligned}
u\left(t, x_{1}, x_{2}\right)= & \int_{0}^{t} d s \int_{\mathbb{R}^{d-1}} G_{1}\left(s, d z_{1}, x_{2}\right) g\left(u\left(t-s, x_{1}-z_{1}, 0\right)\right) \\
& +\int_{[0, t] \times \mathbb{R}^{d-1}} M\left(d s, d z_{1}\right) h\left(u\left(s, z_{1}, 0\right)\right) G_{1}\left(t-s, x_{1}-z_{1}, x_{2}\right),
\end{aligned}
$$

$\mathbb{P}$-a.s., for all $\left(t, x_{1}, x_{2}\right) \in \mathbb{R}_{+} \times \mathbb{R}^{d-1} \times \mathbb{R}$, where $M$ is the worthy martingale measure defined in Section 2.5. The stochastic integral is interpreted as in Section 2.6. A 
jointly measurable adapted process $u=\left\{u(t, x),(t, x) \in \mathbb{R}_{+} \times \mathbb{R}^{d}\right\}$ which satisfies (5.2) is termed a mild solution of equation (5.1).

When $g \equiv 0$ and $h \equiv 1$, the solution of (5.1) is precisely the random-field solution of equation (2.12), which is well defined on $\mathbb{R}_{+} \times \mathbb{R}^{d}$ (see Theorem 4.8) under Assumption $B_{0}^{\prime}$. The following theorem extends this to the non-linear case.

Theorem 5.1. Under Assumptions $B_{0}^{\prime}$ and $C_{0}$, and if $g$ and $h$ are globally Lipschitz functions, then there exists a unique mild solution $u=\left\{u(t, x),(t, x) \in \mathbb{R}_{+} \times \mathbb{R}^{d}\right\}$ to equation (5.1). Moreover, the map $(t, x) \mapsto u(t, x)$ from $\mathbb{R}_{+} \times \mathbb{R}^{d}$ to $L^{2}(\Omega)$ has the continuity property

$$
\lim _{h \downarrow 0, y_{1} \rightarrow x_{1},\left|y_{2}\right| \uparrow\left|x_{2}\right|} E\left(\left(u\left(t+h, y_{1}, y_{2}\right)-u\left(t, x_{1}, x_{2}\right)\right)^{2}\right)=0,
$$

for all $\left(t, x_{1}, x_{2}\right) \in \mathbb{R}_{+} \times \mathbb{R}^{-1} \times \mathbb{R}$. Further, for all $T>0$,

$$
\sup _{(t, x) \in[0, T] \times \mathbb{R}^{d}} \mathbb{E}\left(u(t, x)^{2}\right)<\infty .
$$

We will need the following lemma on the behavior of $\mathcal{F}_{1} G_{1}$.

Lemma 5.2. For all $t>0$, there exists $C(t)>0$ such that for all $s \in[0, t]$ and $\xi_{1} \in \mathbb{R}^{d-1}$,

$$
\left|\mathcal{F}_{1} G_{1}\left(s, \xi_{1}, x_{2}\right)\right| \leq \frac{C(t)}{\left(1+\left|\xi_{1}\right|^{2}\left(s^{2}-x_{2}^{2}\right)\right)^{\frac{1}{4}}} 1_{\left\{\left|x_{2}\right| \leq s\right\}}
$$

Proof of Lemma 5.2. Since $\mathcal{F}_{1} G_{1}\left(s, \xi_{1}, x_{2}\right)=e^{a\left(\sqrt{s^{2}-x_{2}^{2}}-s\right)} \mathcal{F}_{1} G_{1}\left(\sqrt{s^{2}-x_{2}^{2}}, \xi_{1}, 0\right)$, it suffices to prove (5.5) with $x_{2}=0$. In this case, the left-hand side of (5.5) is a continuous function of $\left(s, \xi_{1}\right)$, which is therefore bounded above for $\left|\xi_{1}\right|$ bounded and $s \in[0, t]$. On the other hand, the right-hand side of (5.5) is bounded below for such $\xi_{1}$ and $s$. Therefore, it suffices to check (5.5) for $s \in[0, t]$ and $\left|\xi_{1}\right|$ sufficiently large. In this case, by (3.5) and (C.2),

$$
\begin{aligned}
\left|\mathcal{F}_{1} G_{1}\left(s, \xi_{1}, 0\right)\right| & =\frac{e^{-a s}}{2}\left|J_{0}\left(\sqrt{\left(\left|\xi_{1}\right|^{2}+b-a^{2}\right)} s\right)\right| \\
& \leq \frac{e^{a^{-} t}}{2} \frac{C}{\left(1+\left(\left|\xi_{1}\right|^{2}+b-a^{2}\right) s^{2}\right)^{\frac{1}{4}}} .
\end{aligned}
$$

There is $c>0$ such that for $\left|\xi_{1}\right|$ sufficiently large, $\left|\xi_{1}\right|^{2}+b-a^{2} \geq c\left|\xi_{1}\right|^{2}$, and this leads to (5.5).

With this tool in hand, we can now prove the theorem.

Proof of Theorem [5.1. Let us consider $v\left(t, x_{1}\right)=u\left(t, x_{1}, 0\right),\left(t, x_{1}\right) \in \mathbb{R}_{+} \times \mathbb{R}^{d-1}$. Equation (5.2) evaluated at $x_{2}=0$ gives the following (closed) equation for $v$ :

$$
\begin{aligned}
v\left(t, x_{1}\right)= & \int_{0}^{t} d s \int_{\mathbb{R}^{d-1}} G_{1}\left(s, d z_{1}, 0\right) g\left(v\left(t-s, x_{1}-z_{1}\right)\right) \\
& +\int_{[0, t] \times \mathbb{R}^{d-1}} M\left(d s, d z_{1}\right) h\left(v\left(s, z_{1}\right)\right) G_{1}\left(t-s, x_{1}-z_{1}, 0\right) .
\end{aligned}
$$

Although $G_{1}(\cdot, \cdot, 0)$ is not the Green kernel of any "standard" partial differential equation in $\mathbb{R}_{+} \times \mathbb{R}^{d-1}$, the above equation is of the type of those studied in [5], and we can therefore apply Theorem 13 of that paper. This reference applies (see [6]), since for all $t \in \mathbb{R}_{+}, G_{1}(t, \cdot, 0) \in O_{C}^{\prime}\left(\mathbb{R}^{d-1}\right)_{+}$(by (ii) of Assumption $C_{0}$ ), since 
for all $\xi_{1} \in \mathbb{R}^{d-1}$, the map $t \mapsto \mathcal{F}_{1} G_{1}\left(t, \xi_{1}, 0\right)$ is continuous (by (3.5)) and, finally, since for all $t>0$, there exists $h_{0}>0$ and $k:[0, t] \rightarrow O_{C}^{\prime}\left(\mathbb{R}^{d-1}\right)+$ such that for all $s \in[0, t], h \in\left[0, h_{0}\right]$ and $\xi_{1} \in \mathbb{R}^{d-1}$,

$$
\left|\mathcal{F}_{1} G_{1}\left(s+h, \xi_{1}, 0\right)-\mathcal{F}_{1} G_{1}\left(s, \xi_{1}, 0\right)\right| \leq \mathcal{F}_{1} k\left(s, \xi_{1}\right),
$$

and

$$
\int_{0}^{t} d s \int_{\mathbb{R}^{d-1}} \mu\left(d \xi_{1}\right) \mathcal{F}_{1} k\left(s, \xi_{1}\right)^{2}<\infty
$$

Indeed, by Lemma 5.2 , it suffices to let $k$ be the distribution-valued function whose Fourier transform is given by

$$
\mathcal{F}_{1} k\left(s, \xi_{1}\right)=\frac{2 C(t)}{\left(1+\left(\left|\xi_{1}\right| s\right)^{2}\right)^{\frac{1}{4}}} .
$$

Then (5.7) clearly holds, and for all $s \in[0, t], \mathcal{F}_{1} k(s, \cdot) \in O_{M}\left(\mathbb{R}^{d-1}\right)$, so $k(s, \cdot) \in$ $O_{C}^{\prime}\left(\mathbb{R}^{d-1}\right)$ by (2.3). Moreover, $k(s, \cdot)$ is a non-negative distribution on $\mathbb{R}^{d-1}$ since when $s=0, k(s, \cdot)=2 C(t) \delta_{0}(\cdot)$ which is non-negative, and when $s>0$, by (A.2),

$$
k\left(s, x_{1}\right)=\tilde{C}(t) s^{(1-2 d) / 4}\left|x_{1}\right|^{(3-2 d) / 4} K_{(2 d-3) / 4}\left(\frac{\left|x_{1}\right|}{s}\right), \quad d \in\{2,3\},
$$

where $K_{\nu}$ is the modified Bessel function of order $\nu$ of the second kind, which is non-negative on $\mathbb{R}_{+}$(see Appendix $\mathbf{C}$ ). By using formula (3.11), we obtain that

$$
\begin{aligned}
\int_{0}^{t} d s \int_{\mathbb{R}^{d-1}} \mu\left(d \xi_{1}\right) \mathcal{F}_{1} k\left(s, \xi_{1}\right)^{2} & =\int_{0}^{t} d s \int_{\mathbb{R}^{d-1}} \mu\left(d \xi_{1}\right) \frac{4 C(t)^{2}}{\sqrt{1+\left(\left|\xi_{1}\right| s\right)^{2}}} \\
& \leq \tilde{C}(t) \int_{\mathbb{R}^{d-1}} \mu\left(d \xi_{1}\right) \frac{1+\ln \left(1+\left|\xi_{1}\right|^{2}\right)}{\sqrt{1+\left|\xi_{1}\right|^{2}}}
\end{aligned}
$$

which is finite by Assumption $B_{0}^{\prime}$. Therefore, (5.8) is proved.

Theorem 13 of [5] now states that there exists a unique jointly measurable adapted process $v$ which satisfies (5.6). Moreover, the law of $v\left(t, x_{1}\right)$ is stationary in $x_{1}$ and the map $\left(t, x_{1}\right) \mapsto v\left(t, x_{1}\right)$ from $\mathbb{R}_{+} \times \mathbb{R}^{d-1}$ to $L^{2}(\Omega)$ is right-continuous in $t$ and continuous in $x_{1}$ (note that the right-continuity in $t$ is uniform in $x_{1} \in \mathbb{R}^{d-1}$ ). Further, for all $T>0$,

$$
\sup _{\left(t, x_{1}\right) \in[0, T] \times \mathbb{R}^{d-1}} \mathbb{E}\left(v\left(t, x_{1}\right)^{2}\right)<\infty .
$$

It follows that $u\left(t, x_{1}, 0\right)=v\left(t, x_{1}\right)$ gives the solution of equation (5.2) on the hyperplane $x_{2}=0$. For $x_{2} \neq 0$, let us now define $u\left(t, x_{1}, x_{2}\right)$ by

$$
\begin{aligned}
u\left(t, x_{1}, x_{2}\right)=\int_{0}^{t} d s \int_{\mathbb{R}^{d-1}} G_{1}\left(s, d z_{1}, x_{2}\right) g(v(t & \left.\left.-s, x_{1}-z_{1}\right)\right) \\
& +\left(G_{1}\left(t-\cdot, x_{1}-\cdot, x_{2}\right) \cdot M^{h(v)}\right)_{t},
\end{aligned}
$$

which is not anymore an equation, since $\left(v\left(t, x_{1}\right)\right)$ is now a given process (note that since $G_{1}\left(t-\cdot, x_{1}-\cdot, x_{2}\right)$ is non-negative, $\left\|G_{1}\left(t-\cdot, x_{1}-\cdot, x_{2}\right)\right\|_{t}<\infty$ and $Z=h(v)$ satisfies conditions (2.15) and (2.17), the stochastic integral is well defined by Theorem 2.2).

With $u$ so defined, it is clear that $u$ satisfies equation (5.2) and that it is the unique process to do so. Moreover, it satisfies (5.4) and admits a jointly measurable modification since it satisfies the continuity condition (5.3), as we now prove. 
To this end, write

$$
u\left(t, x_{1}, x_{2}\right)=A\left(t, x_{1}, x_{2}\right)+B\left(t, x_{1}, x_{2}\right),
$$

where

$$
A\left(t, x_{1}, x_{2}\right)=\int_{0}^{t} d s \int_{\mathbb{R}^{d-1}} G\left(s, d z_{1}, x_{2}\right) g\left(v\left(t-s, x_{1}-z_{1}\right)\right)
$$

and

$$
B\left(t, x_{1}, x_{2}\right)=\left(G\left(t-\cdot, x_{1}-\cdot, x_{2}\right) \cdot M^{h(v)}\right)_{t} .
$$

We first verify the $L^{2}$-continuity property (5.3) for the process $B$. Notice that for $x_{2}=0$, this reduces to the continuity property of $v\left(t, x_{1}\right)$, which has already been established. For $x_{2} \neq 0$, we assume without loss of generality that $x_{2}>0$, and fix $\left(t, x_{1}, x_{2}\right) \in \mathbb{R}_{+} \times \mathbb{R}^{d-1} \times \mathbb{R}$ such that $x_{2}<t$. According to the formulas in Appendix $B$,

$$
B\left(t, x_{1}, x_{2}\right)=\int_{0}^{t-x_{2}} \int_{\mathbb{R}^{d-1}} M\left(d s, d z_{1}\right) h\left(v\left(s, z_{1}\right)\right) G_{1}\left(\sqrt{(t-s)^{2}-x_{2}^{2}}, x_{1}-z_{1}, 0\right) .
$$

Therefore, for $h>0$ and $0<y_{2}<x_{2}$,

$$
B\left(t+h, x_{1}, y_{2}\right)-B\left(t, x_{1}, x_{2}\right)=B_{1}+B_{2}
$$

where

$$
\begin{gathered}
B_{1}=\int_{0}^{t-x_{2}} \int_{\mathbb{R}^{d-1}} M\left(d s, d z_{1}\right) h\left(v\left(s, z_{1}\right)\right)\left(G_{1}\left(\sqrt{(t+h-s)^{2}-y_{2}^{2}}, x_{1}-z_{1}, 0\right)\right. \\
\left.-G_{1}\left(\sqrt{(t-s)^{2}-x_{2}^{2}}, x_{1}-z_{1}, 0\right)\right), \\
B_{2}=\int_{t-x_{2}}^{t+h-y_{2}} \int_{\mathbb{R}^{d-1}} M\left(d s, d z_{1}\right) h\left(v\left(s, z_{1}\right)\right) G_{1}\left(\sqrt{(t+h-s)^{2}-y_{2}^{2}}, x_{1}-z_{1}, 0\right) .
\end{gathered}
$$

By (2.19),

$$
E\left(B_{1}^{2}\right)=\int_{0}^{t-x_{2}} d s \int_{\mathbb{R}^{d-1}} \mu_{s}^{h(v)}\left(d \xi_{1}\right) \mid \mathcal{F}_{1} G_{1}\left(\sqrt{(t+h-s)^{2}-y_{2}^{2}},-\xi_{1}, 0\right)
$$

$$
\begin{aligned}
E\left(B_{2}^{2}\right)=\int_{0}^{t} d s \int_{\mathbb{R}^{d-1}} \mu_{s}^{h(v)}\left(d \xi_{1}\right) 1_{\left\{t-x_{2}<s<t+h-y_{2}\right\}} \\
\cdot\left|\mathcal{F}_{1} G_{1}\left(\sqrt{(t+h-s)^{2}-y_{2}^{2}},-\xi_{1}, 0\right)\right|^{2} .
\end{aligned}
$$

The integrand in (5.11) converges pointwise to 0 as $h \downarrow 0, y_{2} \uparrow x_{2}$, and, according to (5.7), is bounded above by $\mathcal{F}_{1} k\left(\sqrt{(t-s)^{2}-x_{2}^{2}},-\xi_{1}\right)$. Since $k \geq 0$, the inequality 
in Theorem 2.2 is valid with $\phi$ replaced by $k$, so we conclude that

$$
\begin{aligned}
& \int_{0}^{t-x_{2}} d s \int_{\mathbb{R}^{d-1}} \mu_{s}^{h(v)}\left(d \xi_{1}\right) \mathcal{F}_{1} k\left(\sqrt{(t-s)^{2}-x_{2}^{2}},-\xi_{1}\right)^{2} \\
& \leq C \int_{0}^{t-x_{2}} d s \int_{\mathbb{R}^{d-1}} \mu\left(d \xi_{1}\right) \mathcal{F}_{1} k\left(\sqrt{(t-s)^{2}-x_{2}^{2}},-\xi_{1}\right)^{2} .
\end{aligned}
$$

Now proceed as in (5.9) to conclude, by Assumption $B_{0}^{\prime}$, that the right-hand side is finite. Therefore, the dominated convergence theorem applies, and we conclude that $E\left(B_{1}^{2}\right) \rightarrow 0$ as $h \downarrow 0$ and $y_{2} \uparrow x_{2}$. The convergence is uniform in $x_{1}$ since the right-hand side of (5.11) does not depend on $x_{1}$.

Since $G_{1}$ is a non-negative distribution, a similar dominated convergence argument shows that $E\left(B_{2}^{2}\right) \rightarrow 0$ uniformly in $x_{1}$ as $h \downarrow 0$ and $y_{2} \uparrow x_{2}$. Therefore, $B\left(t+h, x_{1}, y_{2}\right) \rightarrow B\left(t, x_{1}, x_{2}\right)$ in $L^{2}$, uniformly in $x_{1}$, as $h \downarrow 0$ and $y_{2} \uparrow x_{2}$.

In order to check (5.3) for the process $B$, it remains to show that for $\left(t, x_{2}\right)$ fixed, $B\left(t, y_{1}, x_{2}\right) \rightarrow B\left(t, x_{1}, x_{2}\right)$ in $L^{2}$ as $y_{1} \rightarrow x_{1}$. By (2.19),

$$
\begin{aligned}
& E\left(\left(B\left(t, y_{1}, x_{2}\right)-B\left(t, x_{1}, x_{2}\right)\right)^{2}\right) \\
& \quad=\int_{0}^{t} d s \int_{\mathbb{R}^{d-1}} \mu_{s}^{h(v)}\left(d \xi_{1}\right)\left|\mathcal{F}_{1} G_{1}\left(t-s,-\xi_{1}, x_{2}\right)\right|^{2} \cdot\left|1-e^{i \xi_{1}\left(y_{1}-x_{1}\right)}\right|^{2} .
\end{aligned}
$$

The integrand in (5.13) converges to 0 pointwise as $y_{1} \rightarrow x_{1}$ and is bounded above by $4\left|\mathcal{F}_{1} G_{1}\left(t-s,-\xi_{1}, x_{2}\right)\right|^{2}$. Since $G_{1}$ is a non-negative distribution, we use the inequality in Theorem 2.2 and (5.10) to conclude that

$$
\begin{aligned}
& \int_{0}^{t} d s \int_{\mathbb{R}^{d-1}} \mu_{s}^{h(v)}\left(d \xi_{1}\right) \mid \mathcal{F}_{1} G_{1}(t\left.-s,-\xi_{1}, x_{2}\right)\left.\right|^{2} \\
& \leq C \int_{0}^{t} d s \int_{\mathbb{R}^{d-1}} \mu\left(d \xi_{1}\right)\left|\mathcal{F}_{1} G_{1}\left(t-s,-\xi_{1}, x_{2}\right)\right|^{2} .
\end{aligned}
$$

By Fubini's theorem, Lemma 3.4(b) and Assumption $B_{0}^{\prime}$, the right-hand side is finite. By the dominated convergence theorem, we conclude that the right-hand side of (5.13) converges to 0 as $y_{1} \rightarrow x_{1}$. This completes the proof of (5.3) for the process $B$.

We now verify the $L^{2}$-continuity property of the process $A$. Because of the Lipschitz property of $g,(5.10)$ and the $L^{2}$-continuity property of $v$, there is no special difficulty in checking that for fixed $\left(t, x_{1}, x_{2}\right), A\left(t+h, y_{1}, x_{2}\right)$ converges in $L^{2}$ to $A\left(t, x_{1}, x_{2}\right)$, uniformly in $x_{2}$, as $h \downarrow 0$ and $y_{1} \rightarrow x_{1}$. For fixed $\left(t, x_{1}, x_{2}\right)$, the $L^{2}$-convergence of $A\left(t, x_{1}, y_{2}\right)$ to $A\left(t, x_{1}, x_{2}\right)$ as $y_{2} \rightarrow x_{2}$ is more delicate, since the variable $y_{2}$ appears via the measure $G\left(s, d z_{1}, y_{2}\right)$. To handle this problem, notice by (B.1) $-($ B.3 $)$ that

$$
\begin{gathered}
A\left(t, x_{1}, y_{2}\right)=\int_{\left|y_{2}\right|}^{t} d s e^{a\left(\sqrt{s^{2}-x_{2}^{2}}-s\right)} \int_{\mathbb{R}^{d-1}} G\left(\sqrt{s^{2}-x_{2}^{2}}, d z_{1}, 0\right) g\left(v\left(t-s, x_{1}-z_{1}\right)\right) \\
=\int_{\left|y_{2}\right|}^{t} d s e^{-a s}\left(s^{2}-x_{2}^{2}\right)^{(d-2) / 2} \int_{\mathbb{R}^{d-1}} \nu^{(d)}\left(d y_{1}\right) g^{(d)}\left(\sqrt{s^{2}-x_{2}^{2}}, y_{1}\right) \\
\cdot g\left(v\left(t-s, \sqrt{x^{2}-x_{2}^{2}}\left(y_{1}-z_{1}\right)\right)\right),
\end{gathered}
$$


where $\nu^{(d)}$ and $g^{(d)}$ are defined in Appendix B. In this expression, the measure $\nu^{(d)}$ no longer depends on $x_{2}$. Therefore, the continuity property of $g^{(d)}$, the Lipschitz property of $g$ and the $L^{2}$-continuity properties of $v$ lead, via technical but straightforward calculations, to the desired $L^{2}$-continuity property of $y_{2} \mapsto A\left(t, x_{1}, y_{2}\right)$. 5.1 .

This completes the proof of property (5.3) for $u$, and hence the proof of Theorem

Remark 5.3. As in [5], our proof of Theorem 5.1 applies to equation (5.1) with non-vanishing initial conditions, provided they are stationary in $x_{1}$.

\section{Appendix A. Reformulation of the COndition \\ ON THE SPECTRAL MEASURE}

In this appendix, we reformulate Assumption $B_{0}$ of Section 4.1 into an explicit condition on the covariance of the noise (as has been done in [17, 27]). More generally, consider the condition

$$
\int_{\mathbb{R}^{d-1}} \frac{\mu\left(d \xi_{1}\right)}{\left(1+\left|\xi_{1}\right|^{2}\right)^{\eta}}<\infty
$$

where $\eta>0$ and $d \geq 2$, so Assumption $B_{0}$ corresponds to the case $\eta=\frac{1}{2}$.

Set

$$
G_{d-1, \eta}\left(x_{1}\right)=\mathcal{F}_{1}^{-1}\left(\frac{1}{\left(1+\left|\xi_{1}\right|^{2}\right)^{\eta}}\right)\left(x_{1}\right), \quad x_{1} \in \mathbb{R}^{d-1} .
$$

By [24, formula I.2.7] and a (somewhat tedious) iterative application of [24, formula I.18.29],

$$
G_{d-1, \eta}\left(x_{1}\right)=C_{d}\left|x_{1}\right|^{\eta-\frac{d-1}{2}} K_{\frac{d-1}{2}-\eta}\left(\left|x_{1}\right|\right),
$$

where $K_{\nu}$ is the modified Bessel function of the second kind and of order $\nu$ defined in Appendix [C (this formula appears in [12, Section 56]). If $\eta \leq(d-1) / 2$, then, for definiteness, we set $G_{d-1, \eta}(0)=+\infty$. Let us moreover define the (non-negative) function

$$
H_{d-1, \eta}\left(y_{1}\right)=\int_{\mathbb{R}^{d-1}} \Gamma\left(d x_{1}\right) G_{d-1, \eta}\left(x_{1}-y_{1}\right), \quad y_{1} \in \mathbb{R}^{d-1} .
$$

Proposition A.1. Assume that the covariance measure $\Gamma$ is non-negative on $\mathbb{R}^{d-1}$. Then condition (A.1) holds if and only if

$$
\sup _{y_{1} \in \mathbb{R}^{d-1}} H_{d-1, \eta}\left(y_{1}\right)<\infty .
$$

Remark A.2. Note that (A.3) implies that

$$
H_{d-1, \eta}(0)=\int_{\mathbb{R}^{d-1}} \Gamma\left(d x_{1}\right) G_{d-1, \eta}\left(x_{1}\right)<\infty
$$


which, because of the exponential decrease of $G_{d-1, \eta}\left(x_{1}\right)$ as $\left|x_{1}\right| \rightarrow \infty$ (see (C.6)), can be reformulated in turn (see formula (C.7)) as

$$
\begin{cases}\text { no particular condition on } \Gamma, & \text { when } \eta>\frac{d-1}{2}, \\ \int_{B_{d-1}(0,1)} \Gamma\left(d x_{1}\right) \ln \left(\frac{1}{\left|x_{1}\right|}\right)<\infty, & \text { when } \eta=\frac{d-1}{2}, \\ \int_{B_{d-1}(0,1)} \Gamma\left(d x_{1}\right) \frac{1}{\left|x_{1}\right|^{d-1-2 \eta}}<\infty, & \text { when } \eta<\frac{d-1}{2} .\end{cases}
$$

On the other hand, note that since $H_{d-1, \eta}$ is non-negative definite, the condition " $H_{d-1, \eta}$ is continuous at 0 " implies (A.3) (see [28, Chap. VII, p. 276]).

Proof of Proposition A.1. Suppose first that condition (A.1) is satisfied. Let $p_{t}=$ $\mathcal{F}_{1}^{-1}\left(e^{-t\left|\xi_{1}\right|^{2}}\right)$ denote the heat kernel in $\mathbb{R}^{d-1}$. Since

$$
\left(G_{d-1, \eta} * p_{t}\right)\left(x_{1}-y_{1}\right) \underset{t \downarrow 0}{\longrightarrow} G_{d-1, \eta}\left(x_{1}-y_{1}\right), \quad \text { for all } x_{1} \in \mathbb{R}^{d-1},
$$

Fatou's lemma implies that

$$
\begin{aligned}
H_{d-1, \eta}\left(y_{1}\right) & =\int_{\mathbb{R}^{d-1}} \Gamma\left(d x_{1}\right) G_{d-1, \eta}\left(x_{1}-y_{1}\right) \\
& \leq \liminf _{t \downarrow 0} \int_{\mathbb{R}^{d-1}} \Gamma\left(d x_{1}\right)\left(G_{d-1, \eta} * p_{t}\right)\left(x_{1}-y_{1}\right) .
\end{aligned}
$$

Now, since

$$
\int_{\mathbb{R}^{d-1}} \Gamma\left(d x_{1}\right)\left(G_{d-1, \eta} * p_{t}\right)\left(x_{1}-y_{1}\right)=\int_{\mathbb{R}^{d-1}} \mu\left(d \xi_{1}\right) \frac{1}{\left(1+\left|\xi_{1}\right|^{2}\right)^{\eta}} e^{-t\left|\xi_{1}\right|^{2}} e^{i \xi_{1} \cdot y_{1}}
$$

and

$$
\left|\int_{\mathbb{R}^{d-1}} \mu\left(d \xi_{1}\right) \frac{1}{\left(1+\left|\xi_{1}\right|^{2}\right)^{\eta}} e^{-t\left|\xi_{1}\right|^{2}} e^{i \xi_{1} \cdot y_{1}}\right| \leq \int_{\mathbb{R}^{d-1}} \mu\left(d \xi_{1}\right) \frac{1}{\left(1+\left|\xi_{1}\right|^{2}\right)^{\eta}}<\infty
$$

by assumption (A.1), we obtain that $H_{d-1, \eta}$ is a bounded function on $\mathbb{R}^{d-1}$.

In order to prove the converse, assume $H_{d-1, \eta}$ is bounded and note that since $p_{t}$ is a probability measure on $\mathbb{R}^{d-1}$ for all $t \in \mathbb{R}_{+}$, we have

$$
\sup _{t \in \mathbb{R}_{+}} \int_{\mathbb{R}^{d-1}} d y_{1} p_{t}\left(y_{1}\right) H_{d-1, \eta}\left(y_{1}\right) \leq \sup _{y_{1} \in \mathbb{R}^{d-1}} H_{d-1, \eta}\left(y_{1}\right)<\infty .
$$

On the other hand,

$$
\int_{\mathbb{R}^{d-1}} d y_{1} p_{t}\left(y_{1}\right) H_{d-1, \eta}\left(y_{1}\right)=\int_{\mathbb{R}^{d-1}} \Gamma\left(d x_{1}\right)\left(G_{d-1, \eta} * p_{t}\right)\left(x_{1}\right),
$$

by definition of $H_{d-1, \eta}$ and Fubini's theorem. By (A.5), this expression is equal to

$$
\int_{\mathbb{R}^{d-1}} \mu\left(d \xi_{1}\right) \frac{1}{\left(1+\left|\xi_{1}\right|^{2}\right)^{\eta}} e^{-t\left|\xi_{1}\right|^{2}}
$$

and this converges by the monotone convergence theorem to

$$
\int_{\mathbb{R}^{d-1}} \mu\left(d \xi_{1}\right) \frac{1}{\left(1+\left|\xi_{1}\right|^{2}\right)^{\eta}}, \quad \text { as } t \downarrow 0 .
$$

The proposition is proved. 


\section{Appendix B. Green Kernel of the hyperbolic EQUation in $\mathbb{R}^{d}$}

When $d \in\{2,3\}$ and $a^{2} \geq b$, we have the following expressions for $G_{1}\left(t, \cdot, x_{2}\right)$ (which can be computed using the expression for $\mathcal{F} G$ and the Fourier inversion formula). Fix $t \in \mathbb{R}_{+}$and $\left.x_{2} \in\right]-t, t[$. For $d=2$, we have

$$
\begin{aligned}
G_{1}\left(t, x_{1}, x_{2}\right) & =\frac{e^{-a t}}{2 \pi} 1_{\left\{\left|x_{2}\right|<t\right\}} \frac{\cosh \left(\sqrt{\left(a^{2}-b\right)\left(t^{2}-x_{2}^{2}-x_{1}^{2}\right)}\right)}{\sqrt{t^{2}-x_{2}^{2}-x_{1}^{2}}} 1_{\left\{\left|x_{1}\right|<\sqrt{t^{2}-x_{2}^{2}}\right\}} \\
& =e^{a\left(\sqrt{t^{2}-x_{2}^{2}}-t\right)} G_{1}\left(\sqrt{t^{2}-x_{2}^{2}}, x_{1}, 0\right),
\end{aligned}
$$

and for $d=3$, we have

$$
\begin{aligned}
G_{1}\left(t, d x_{1}, x_{2}\right)= & \frac{e^{-a t}}{4 \pi} 1_{\left\{\left|x_{2}\right|<t\right\}}\left(\frac{1}{\sqrt{t^{2}-x_{2}^{2}}} \sigma^{(3)}\left(d x_{1}\right) 1_{\partial B\left(0, \sqrt{t^{2}-x_{2}^{2}}\right)}\left(x_{1}\right)\right. \\
& \left.+\sqrt{a^{2}-b} \frac{I_{1}\left(\sqrt{\left(a^{2}-b\right)\left(t^{2}-x_{2}^{2}-\left|x_{1}\right|^{2}\right)}\right)}{\sqrt{t^{2}-x_{2}^{2}-\left|x_{1}\right|^{2}}} 1_{\left\{\left|x_{1}\right|<\sqrt{t^{2}-x_{2}^{2}}\right\}} d x_{1}\right) \\
= & e^{a\left(\sqrt{t^{2}-x_{2}^{2}}-t\right)} G_{1}\left(\sqrt{t^{2}-x_{2}^{2}}, d x_{1}, 0\right),
\end{aligned}
$$

where $I_{1}$ is the first order modified Bessel function of the first kind (see Appendix C), and $\sigma^{(3)}$ is the 2-dimensional Hausdorff measure as in Example 3.2 (for $a=0$, the above formula can be found in [14, formula (7.3.88)]). These two formulas show that for $d \in\{2,3\}, G_{1}\left(t, \cdot, x_{2}\right)$ is a non-negative measure.

Observe in addition that for any Borel function $h: \mathbb{R}^{d-1} \rightarrow \mathbb{R}$,

$$
\int_{\mathbb{R}^{d-1}} G\left(r, d x_{1}, 0\right) h\left(x_{1}\right)=1_{\{r>0\}} r^{d-2} e^{-a r} \int_{\mathbb{R}^{d-1}} \nu^{(d)}\left(d y_{1}\right) g^{(d)}\left(r, y_{1}\right) h\left(r y_{1}\right),
$$

where for $d=2$,

$$
\nu^{(2)}\left(d y_{1}\right)=1_{\left\{\left|y_{1}\right|<1\right\}} \frac{d y_{1}}{\sqrt{1-y_{1}^{2}}}, \quad g^{(2)}\left(r, y_{1}\right)=\cosh \left(r \sqrt{\left(a^{2}-b\right)\left(1-y_{1}^{2}\right)}\right)
$$

and for $d=3$,

$$
\begin{aligned}
\nu^{(3)}\left(d y_{1}\right) & =\sigma^{(3)}\left(d y_{1}\right) 1_{\partial B(0,1)}\left(y_{1}\right)+\sqrt{a^{2}-b} 1_{\left\{\left|y_{1}\right|<1\right\}} \frac{d y_{1}}{\sqrt{1-\left|y_{1}\right|^{2}}}, \\
g^{(3)}\left(r, y_{1}\right) & =I_{1}\left(r \sqrt{\left(a^{2}-b\right)\left(1-\left|y_{1}\right|^{2}\right)}\right) .
\end{aligned}
$$

\section{Appendix C. Bessel functions}

The zero order regular and modified Bessel funtions of the first kind are given respectively (see formulas 9.1 .10 and 9.6.10 in [1]) by

$$
J_{0}(r)=\sum_{n \in \mathbb{N}}(-1)^{n} \frac{\left(\frac{r}{2}\right)^{2 n}}{(n !)^{2}} \quad \text { and } \quad I_{0}(r)=\sum_{n \in \mathbb{N}} \frac{\left(\frac{r}{2}\right)^{2 n}}{(n !)^{2}}, \quad r \in \mathbb{R}_{+},
$$


and we have the following integral representations (see formulas 9.1.18 and 9.6.16 in [1]):

$$
J_{0}(r)=\frac{1}{\pi} \int_{0}^{\pi} \cos (r \sin (t)) d t \quad \text { and } \quad I_{0}(r)=\frac{1}{\pi} \int_{0}^{\pi} \cosh (r \cos (t)) d t, \quad r \in \mathbb{R}_{+} .
$$

Therefore, $J_{0}(0)=1$ and $J_{0}$ is decreasing on $[0,1]$ since $J_{0}^{\prime}(r)<0$ on this interval. Moreover, $\left|J_{0}(r)\right| \leq 1$ for all $r \in \mathbb{R}_{+}$and $J_{0}$ only has a countable set of zeros [1, Sect. 9.5]. On the other hand, $I_{0}(0)=1$ and $I_{0}$ is increasing on $\mathbb{R}_{+}$. By formula 9.2.1 in [1] there exists $C>0$ such that

$$
\left|J_{0}(r)-\sqrt{\frac{2}{\pi r}} \cos \left(r-\frac{\pi}{4}\right)\right| \leq \frac{C}{r^{3 / 2}}, \quad \text { for all } r>0 .
$$

In particular, there exists $C>0$ such that for all $r \geq 0$,

$$
J_{0}(r)^{2} \leq \frac{C}{\sqrt{1+r^{2}}} \leq \frac{C}{r} .
$$

Lemma C.1. There exists $C>0$ such that for all $R \geq 1$,

$$
\frac{1}{R} \int_{0}^{R} d r r J_{0}(r)^{2} \geq C .
$$

Proof. Since the left-hand side of the inequality is a continuous and strictly positive function of $R$ (for $R \neq 0$ ), it suffices to show that

$$
\lim _{R \rightarrow \infty} \frac{1}{R} \int_{0}^{R} d r r J_{0}(r)^{2}=\frac{1}{\pi} .
$$

To see this, note that by (C.1) and (C.2),

$$
\begin{aligned}
\mid r & J_{0}(r)^{2}-\frac{2}{\pi} \cos ^{2}\left(\frac{\pi}{4}-r\right) \mid \\
& =\left|\sqrt{r} J_{0}(r)-\sqrt{\frac{2}{\pi}} \cos \left(\frac{\pi}{4}-r\right)\right|\left|\sqrt{r} J_{0}(r)+\sqrt{\frac{2}{\pi}} \cos \left(\frac{\pi}{4}-r\right)\right| \\
& \leq \frac{C}{1+r}
\end{aligned}
$$

This implies that

$$
\left|\int_{0}^{R} d r r J_{0}(r)^{2}-\frac{2}{\pi} \int_{0}^{R} d r \cos ^{2}\left(\frac{\pi}{4}-r\right)\right| \leq C \ln (1+R) .
$$

Since

$$
\lim _{R \rightarrow \infty} \frac{1}{R} \int_{0}^{R} d r \cos ^{2}\left(\frac{\pi}{4}-r\right)=\frac{1}{2}
$$

(C.3) holds, and this completes the proof.

Lemma C.2. There exists $c>0$ such that for all $R>0$,

$$
\int_{0}^{R} d r J_{0}(r)^{2} \geq c \ln (R) .
$$


Proof. The left-hand side is a positive and continuous function of $R$, while the righthand side is negative for $R<1$ and vanishes at $R=1$. Therefore, it is sufficient to check the inequality for $R$ sufficiently large.

By (C.4), there exists $C>0$ such that for all $r \geq 1$,

$$
\left|J_{0}(r)^{2}-\frac{2}{\pi r} \cos ^{2}\left(\frac{\pi}{4}-r\right)\right| \leq \frac{C}{r^{2}}
$$

Therefore, for $R \geq 2$,

$$
\left|\int_{1}^{R} d r J_{0}(r)^{2}-\frac{2}{\pi} \int_{1}^{R} d r \frac{1}{r} \cos ^{2}\left(\frac{\pi}{4}-r\right)\right| \leq C \int_{1}^{\infty} d r \frac{1}{r^{2}} \leq c_{2}<\infty .
$$

Elementary computations show that for large $R$,

$$
\int_{1}^{R} d r \frac{\cos ^{2}\left(\frac{\pi}{4}-r\right)}{r} \geq \tilde{c}_{1} \ln (R)
$$

and since the expressions on both sides are continuous functions of $R$, we conclude that there is $c_{1}>0$ such that for $R \geq 2$,

$$
\int_{0}^{R} d r J_{0}(r)^{2} \geq \int_{1}^{R} d r J_{0}(r)^{2} \geq c_{1} \ln (R)-c_{2} .
$$

For $R$ sufficiently large, the right-hand side is bounded below by $\frac{1}{2} c_{1} \ln (R)$, and this completes the proof.

The modified Bessel function of first order, denoted $I_{1}$ and appearing in expression (B.2), is defined by $I_{1}(r)=I_{0}^{\prime}(r), r \in \mathbb{R}_{+}$(see formula 9.6.27 in [1]). It is therefore non-negative, since $I_{0}$ is increasing, and $I_{1}(0)=0$.

Finally, for $\nu \in \mathbb{R}_{+}$, the modified Bessel functions of the second kind and of order $\nu$ are given (see formula 9.6.23 in [1]) by

$$
K_{\nu}(r)=\frac{\sqrt{\pi}}{\Gamma\left(\nu+\frac{1}{2}\right)}\left(\frac{r}{2}\right)^{\nu} \int_{1}^{\infty} d t e^{-r t}\left(t^{2}-1\right)^{\nu-\frac{1}{2}}, \quad r \in \mathbb{R}_{+},
$$

where $\Gamma$ is the Euler Gamma function, and $K_{-\nu}(r)=K_{\nu}(r)$ by formula 9.6.6 in [1]. From this definition, we see that $K_{\nu}$ is non-negative, for all $\nu \in \mathbb{R}_{+}$. By formula 9.7.2 in [1, there exists $C>0$ such that

$$
K_{\nu}(r) \leq C e^{-r}, \quad \text { for all } r \geq 1 .
$$

On the other hand, when $r \rightarrow 0$, we have by formulas 9.6.8 and 9.6.9 in [1]:

$$
K_{\nu}(r) \sim\left\{\begin{array}{cc}
\ln \left(\frac{1}{r}\right) & \text { if } \nu=0 \\
r^{-|\nu|} & \text { if } \nu \neq 0
\end{array}\right.
$$




\section{ApPENDix D. InDEX OF NOTATION}

\begin{tabular}{|c|c|}
\hline $\mathcal{B}_{b}\left(\mathbb{R}^{d}\right)$ & set of bounded Borel subsets of $\mathbb{R}^{d}$ \\
\hline$B_{d}(x, r)$ & at $x$ with radius $r$ \\
\hline$\chi_{\xi}(x)$ & $=e^{i \xi \cdot x}, x \in \mathbb{R}^{d}$ \\
\hline & $\begin{array}{l}\text { Dirac measure at } x \in \mathbb{R}^{d} \\
\text { spaces of elementary integrands (see Sections } 2.3 \text { and } 2.5 \text { ) }\end{array}$ \\
\hline $\mathcal{F} T$ & ourier transform of $T \in \mathcal{S}^{\prime}\left(\mathbb{R}^{d}\right)$ (see Section 2.1) \\
\hline $\mathcal{F}_{1} T, \mathcal{F}_{2} T$ & ourier transforms of $T$ in $x_{1}, x_{2}$ (see Section 2.4 $)$ \\
\hline$\dot{F}\left(t, x_{1}\right)$ & Gaussian noise on the hyperplane $\mathbb{R}^{d-1} \times\{0\}$ (see (2.12) \\
\hline$G(t, \cdot), G_{1}\left(t, \cdot, x_{2}\right)$ & $\begin{array}{l}\text { Green's function and its restriction to a hyperplane } \\
\text { (see Sections 3 3.2) }\end{array}$ \\
\hline$\Gamma_{0}, \nu_{0}$ & leasures on $\mathbb{R}^{d} \times \mathbb{R}^{d}$ (see Section 2.2 ) \\
\hline$\Gamma\left(d x_{1}\right), \Gamma_{s}^{Z}\left(d x_{1}\right)$ & 2.4 and 2.6 \\
\hline$H_{t,+, 0}, H_{t,+}$ & $\begin{array}{l}\text { oaces of Borel-measurable integrands (see Sections } 2.3 \\
\text { ad 2.5) }\end{array}$ \\
\hline$H_{t,+, Z}, H_{t, 0}$ & ces of Borel-measurable integrands (see Section 2.6) \\
\hline$H_{t, Z}, H_{t}$ & $n[2.6)$ \\
\hline$J_{0}, K_{0}, K_{\nu}$ & Bessel functions (see Appendix C) \\
\hline$M^{0}, M, M^{Z}$ & $\begin{array}{l}\text { worthy martingale measures (see Sections } 2.3,2.5 \\
\text { and } 2.6 \text { ) }\end{array}$ \\
\hline$\mu\left(d \xi_{1}\right), \mu_{s}^{Z}\left(d \xi_{1}\right)$ & measures (see Sections 2.4 and 2.6) \\
\hline$\nu\left(d x_{1}\right)$ & domi \\
\hline$O_{M}\left(\mathbb{R}^{d}\right)$ & functions on $\mathbb{R}^{d}$ with \\
\hline$O_{C}^{\prime}\left(\mathbb{R}^{d}\right)$ & space of distributions with rapid decrease on $\mathbb{R}^{d}$ \\
\hline & ble $\sigma$-fields (se \\
\hline $\mathcal{S}\left(\mathbb{R}^{d}\right)$ & functions on $\mathbb{R}^{d}$ with rapid \\
\hline $\mathcal{S}^{\prime}\left(\mathbb{R}^{d}\right)$ & tempered distributions on $\mathbb{R}^{d}$ \\
\hline$x=\left(x_{1}, x_{2}\right)$ & $\begin{array}{l}x_{1} \text { represents the first } d-1 \text { coordinates of } x \in \mathbb{R}^{d} \text {, } \\
x_{2} \text { the last coordinate }\end{array}$ \\
\hline & predictable $\mathrm{p}$ \\
\hline & semi-inner product and norm on $H_{t, 0}$ (see Section 2.3) \\
\hline & $\begin{array}{l}\text { semi-inner products on } H_{t, Z}, H_{t} \text { (see Sections 2.6 } \\
\text { and 2.5) }\end{array}$ \\
\hline & semi-norms on $H_{t, Z}, H_{t}$ (see Sections 2.6 and 2.5) \\
\hline & $\begin{array}{l}\text { semi-norms on } H_{t,+, 0} \text { and } H_{t,+, Z} \text { (see Sections } 2.3 \\
\text { and 2.6) }\end{array}$ \\
\hline 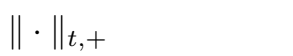 & on $H_{t,+}$ (see Section 2.5) \\
\hline
\end{tabular}

\section{REFERENCES}

[1] Abramowitz M., Stegun I.A., "Handbook of mathematical functions", 1964, National Bureau of Standards. MR0167642 (29:4914)

[2] Alòs E., Bonnacorsi S., "Stochastic partial differential equations with Dirichlet white-noise boundary conditions", Ann. Inst. H. Poincaré Probab. Statist. 38(2), 2002, pp. 125-154. MR 1899108 (2003a:60093)

[3] Badii L., Oberhettinger F., "Tables of Laplace transforms", 1973, Springer Verlag. MR 0352889(50:5375) 
[4] Berg C., Christensen J.P.R., Ressel P., "Harmonic analysis on semigroups", 1984, Springer Verlag. MR0747302 (86b:43001)

[5] Dalang R.C., "Extending the martingale measure stochastic integral with applications to spatially homogeneous SPDE's", Electronic Journal of Probability, Vol. 4, 1999, Article No. 6. MR1684157 (2000b:60132)

[6] Dalang R.C., "Corrections to Extending the martingale measure stochastic integral with applications to spatially homogeneous SPDE's", Electronic Journal of Probability, Vol. 6, 2001. MR 1825714 (2002b:60111)

[7] Dalang R.C., Frangos N.E., "The stochastic wave equation in two spatial dimensions", Ann. Prob., Vol. 26 (1), 1998, pp. 187-212. MR1617046 (99c:60127)

[8] Dawson D.A., Salehi H., "Spatially homogeneous random evolutions", J. Mult. Anal., Vol. 10, 1980, pp. 141-180. MR0575923 (82c:60102)

[9] Da Prato G., Zabczyck J., "Stochastic equations in infinite dimensions", Encyclopedia of Mathematics and its Applications 44, 1992, Cambridge University Press. MR 1207136 (95g:60073)

[10] Da Prato G., Zabczyck J., "Evolution equations with white-noise boundary conditions", Stoch. and Stoch. Reports, Vol. 42, 1993, pp. 167-182. MR.1291187 (95m:60088)

[11] De Brucq D., Olivier, C., "Approximations des processus gaussiens stationaires, solutions d'équations aux dérivées partielles linéaires", Rev. Roum. Math. Pures et Appl., Tome XXVIII, 1983, pp. 205-228. MR0705453 (84m:60050)

[12] Donoghue W.F., "Distributions and Fourier Transforms", 1969, Academic Press.

[13] Durrett R., "Probability: theory and examples", 1996, Duxbury Press. MR.1609153 (98m:60001)

[14] Feshbach H., Morse P.M., "Methods in theoretical physics", Vol. I and II, 1953, McGraw Hill. MR0059774 (15:583h)

[15] Folland G. B., "Introduction to partial differential equations", 1995, Princeton University Press. MR:1357411 (96h:35001)

[16] Hörmander L., "The analysis of linear partial differential operators", Vol. 2, 1984, Springer Verlag. MR.0705278 (85g:35002b)

[17] Karczewska A., Zabczyck J., "Stochastic PDE's with function-valued solutions", in Clément Ph., den Hollander F., van Neerven J. and de Pagter B. (Eds), "Infinite-dimensional stochastic analysis", Proceedings of the Colloquium of the Royal Netherlands Academy of Arts and Sciences, 1999, Amsterdam. MR1832378 (2002h:60132)

[18] Mao X., Markus L., "Wave equation with stochastic boundary values", J. Math. Anal. and Appl., Vol. 177, 1993, pp. 315-341. MR1231485 (94g:60115)

[19] Maslowski B., "Stability of semilinear equations with boundary and pointwise noise", Annali della Scuola Normale Superiore di Pisa, Serie IV, Vol. XXII, 1995, Fasc. 1. MR1315350 (95m:35214)

[20] Millet A., Sanz-Solé M., "A stochastic wave equation in two space dimension: smoothness of the law" Ann. Prob., Vol. 27 (2), 1999, pp. 803-844. MR1698971 (2001e:60130)

[21] Millet A., Sanz-Solé M., "Approximation and support theorem for a wave equation in two space dimensions", Bernoulli, Vol. 6 (5), 2000, pp. 887-915. MR:1791907 (2001m:60147)

[22] Mueller C., "Long time existence for the wave equation with a noise term", Ann. Prob., Vol. 25(1), 1997, pp.133-151. MR.1428503 (98b:60113)

[23] Neveu J., "Processus aléatoires gaussiens", 1968, Presses de l'Université de Montréal. MR.0272042 (42:6923)

[24] Oberhettinger F., "Tables of Fourier transforms and Fourier transforms of distributions", 1990, Springer Verlag. MR1055360 (91g:00010)

[25] Peszat S., "The Cauchy problem for a nonlinear stochastic wave equation in any dimension", J. Evol. Eq., Vol. 2, 2002, pp. 383-394. MR1930613 (2003k:60157)

[26] Peszat S., Zabczyck J., "Nonlinear stochastic wave and heat equations", Prob. Th. and Rel. Fields, Vol. 116, 2000, pp. 421-443. MR1749283(2001f:60071)

[27] Sanz-Solé M., Sarrà M., "Path properties of a class of Gaussian processes with applications to SPDE's", in: Stochastic processes, physics and geometry: new interplays, I (Leipzig, 1999), CMS Conf. Proc., Vol. 28, 2000, Amer. Math. Soc., Providence, RI, pp. 303-316. MR 1803395 (2001m:60148)

[28] Schwartz L., "Théorie des distributions", 1966, Hermann. MR0209834 (35:730) 
[29] Sowers R., "Multidimensional reaction-diffusion equations with white noise boundary perturbations", Ann. of Prob., Vol. 22 (4), 1994, pp. 2071-2121. MR1331216 (96k:60161)

[30] Walsh J.B., "An introduction to stochastic partial differential equations", Ecole d'été de probabilités de Saint-Flour XIV, Lecture Notes in Math., 1984, Springer Verlag. MR0876085 (88a:60114)

[31] Wheeden R.L., Zygmund A., "Measure and integral: an introduction to real analysis", 1977, Marcel Dekker. MR0492146 (58:11295)

[32] Wilcox C.H., "The Cauchy problem for the wave equation with distribution data: an elementary approach", The American Mathematical Society Monthly, Vol. 98, 1991, pp. 401-410. MR.1104303 (92h:35227)

Institut de Mathématiques, Ecole Polytechnique Fédérale, Station 8, 1015 Lausanne, SWITZERLAND

E-mail address: robert.dalang@epfl.ch

Institut de Systèmes de Communication, Ecole Polytechnique Fédérale, Station 14, 1015 Lausanne, Switzerland

E-mail address: olivier.leveque@epfl.ch 